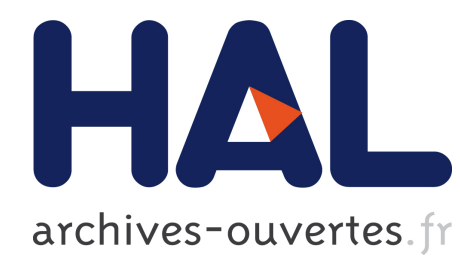

\title{
Mechanics-Aided Digital Image Correlation
}

\author{
Zvonimir Tomicevic, François Hild, Stéphane Roux
}

\section{- To cite this version:}

Zvonimir Tomicevic, François Hild, Stéphane Roux. Mechanics-Aided Digital Image Correlation. Journal of Strain Analysis for Engineering Design, SAGE Publications, 2013, 48, pp.330343. $<10.1177 / 0309324713482457>$. <hal-00848729>

\section{HAL Id: hal-00848729 \\ https://hal.archives-ouvertes.fr/hal-00848729}

Submitted on 28 Jul 2013

HAL is a multi-disciplinary open access archive for the deposit and dissemination of scientific research documents, whether they are published or not. The documents may come from teaching and research institutions in France or abroad, or from public or private research centers.
L'archive ouverte pluridisciplinaire HAL, est destinée au dépôt et à la diffusion de documents scientifiques de niveau recherche, publiés ou non, émanant des établissements d'enseignement et de recherche français ou étrangers, des laboratoires publics ou privés. 


\title{
Mechanics-Aided Digital Image Correlation
}

\author{
Zvonimir Tomičević, ${ }^{1,2}$ François Hild ${ }^{2, *}$ and Stéphane Roux ${ }^{2}$ \\ ${ }^{1}$ Department of Engineering Mechanics \\ Faculty of Mechanical Engineering and Naval Architecture \\ University of Zagreb, Ivana Lučića 5, 10000 Zagreb, Croatia \\ ${ }^{2}$ LMT-Cachan, ENS Cachan/CNRS/UPMC/PRES UniverSud Paris \\ 61 avenue du Président Wilson, F-94235 Cachan Cedex, France
}

\begin{abstract}
By construction digital image correlation is an ill-posed problem. To circumvent this difficulty, the regularization is often performed implicitly through the kinematic basis chosen to express the sought displacement fields. Conversely, a priori information on the mechanical behavior of the studied material is often available. It is proposed to evaluate the gain to be expected from such a mechanical assistance, namely, the measured displacement not only satisfies as best as possible the gray level conservation, but also mechanical admissibility.
\end{abstract}

Keywords: Equilibrium gap; Full-field measurements; Regularization; Resolution; T3 elements.

${ }^{*}$ Corresponding author, email: hild@lmt.ens-cachan.fr 


\section{Introduction}

One of the limitations of Digital Image Correlation (DIC) comes from its ill-posedness. The limited available information, generally two gray-level images to be registered, impedes the measurement of very rapid fluctuations of the displacement field, and hence constitutes a potential barrier to very small scale displacement resolutions (i.e., the 'smallest change in a quantity being measured that causes a perceptible change in the corresponding indication' [1]). Consequently, a compromise has to be found between the standard measurement uncertainty and the spatial resolution $[2,3]$.

Different strategies have been designed to overcome this limitation. The most classical one is to work with a coarse description of the displacement field based on a discretization scale that is much larger than the pixel scale. This corresponds to the choice made in local (or subset-based) DIC approaches $[4,5,6]$ where the size of the Zone Of Interest (ZOI) can be seen as a natural regularization. Apart from this coarse description, no additional assumption is made on the displacement field, which finally has to be interpolated between ZOI centers. In practice, this choice limits the ZOI size to typically 10 pixels or more $[7,8]$.

To achieve smaller resolutions or spatial resolutions, some additional information has to be provided. One example is to assume that the displacement field be continuous. The latter can be decomposed over a convenient basis of functions that fulfill this constraint. For instance, a finite element

basis can be chosen $[9,10,11]$. The problem to solve no longer consists in a collection of independent correlation computation for each ZOI as was the 
case for local DIC [8]. The problem is formulated as a whole and all degrees of freedom determined simultaneously through the solution of a coupled problem, it is referred to as 'global DIC.' This coupling involves an over-cost in terms of computation time. This extra cost is however rewarding in terms of result quality [12]. Further, the post-processing step of data interpolation and smoothing becomes essentially useless, and hence a good control of the displacement determination is preserved. In favorable cases, element sizes as small as $4 \times 4$ pixels can be used [13].

The spatial resolution can be further decreased (i.e., down to pixel [14] or voxel scales $[15,16])$ by adding other terms in the minimization of the gray level conservation [17]. A natural choice stems from the general propositions associated with the regularization of ill-posed problems [18]. It consists in minimizing the fluctuations associated with the Laplacian of the displacement field [14]. This type of filter does work, but it may discard fluctuations that are mechanically admissible.

Another route consists in considering a mechanical filter, namely, the regularization term allows the mechanical admissibility to be enforced in a weak sense. Among the various propositions [19], the equilibrium gap is chosen $[20,17]$ in the sequel. The advantage of such a regularization will be shown when assessing the resolution, and with very difficult textures that are not tractable with classical DIC codes. Further, boundary regularization has not received a lot attention. When comparing experimental and simulated displacement fields for identification or validation purposes, the raw measured boundary conditions are considered [21] or filtered [22]. They can also be parts of the unknowns in an integrated approach to DIC [23]. A new 
mechanics-based regularization is proposed herein. It acts as a filter in the same spirit as for bulk degrees of freedom. In the following studies, 3-noded elements will be considered. They are the simplest in terms of displacement interpolations, even though they are seldom used, if ever, in the framework of global DIC. The other novelty of the developed procedure is that the resolution / spatial resolution (or regularization length) limit can be broken through an appropriate driving of the DIC algorithm.

The paper is organized as follows. First, the main features associated with global DIC and its regularization are introduced in Section 2. Section 3 is devoted to the analysis of two artificial cases. The first one deals with a sinusoidal displacement field, and the second one with a mechanically admissible field. True experimental data are finally studied in Section 4. Natural and artificial textures are compared.

\section{Regularized Digital Image Correlation}

\section{$2.1 \quad$ Regularization Strategy}

The registration of two gray level pictures in the reference, $f$, and deformed, $g$, configurations is based upon the conservation of the gray levels

$$
f(\mathbf{x})=g(\mathbf{x}+\mathbf{u}(\mathbf{x}))
$$

where $\mathbf{u}$ is the unknown displacement field to be measured, and $\mathbf{x}$ the pixel location. The sought displacement field minimizes the sum of squared differ- 
ences $\Phi_{c}^{2}$ over the Region Of Interest (ROI)

$$
\Phi_{c}^{2}=\int_{\mathrm{ROI}} \varphi_{c}^{2}(\mathbf{x}) \mathrm{d} \mathbf{x}
$$

where $\varphi_{c}(\mathbf{x})$ defines the field of correlation residuals

$$
\varphi_{c}(\mathbf{x})=|g(\mathbf{x}+\mathbf{u}(\mathbf{x}))-f(\mathbf{x})|
$$

The minimization of $\Phi_{c}^{2}$ is a nonlinear and ill-posed problem. If no additional information is available, it is impossible to determine the displacement for each pixel independently since there are two unknowns for a given (scalar) gray level difference. This explains the reason for choosing a weak formulation in which the displacement field is expressed in a (chosen) basis as

$$
\mathbf{u}(\mathbf{x})=\sum_{n} u_{n} \boldsymbol{\psi}_{n}(\mathbf{x})
$$

where $\boldsymbol{\psi}_{n}$ are (chosen) vector fields, and $u_{n}$ the associated degrees of freedom. The measurement problem then consists in minimizing $\Phi_{c}^{2}$ with respect to the unknowns $u_{n}$. A Newton iterative procedure is followed to circumvent the nonlinear aspect of the minimization problem [11]. The following linear systems are solved iteratively

$$
[\mathbf{M}]\{\delta \mathbf{u}\}=\{\mathbf{b}\}
$$

where $[\mathbf{M}]$ is a matrix formed from the dyadic product of fields $\nabla f \cdot \boldsymbol{\psi}_{n}[12]$, $\{\mathbf{b}\}$ a vector that vanishes when a perfect registration is obtained for each pixel (i.e., Equation (1)) is satisfied), and $\{\delta \mathbf{u}\}$ collects the corrections to the measured degrees of freedom $u_{n}$.

To enforce mechanical admissibility in an FE sense, the equilibrium gap is first introduced [20]. If linear elasticity applies, the equilibrium equations 
read

$$
[\mathbf{K}]\{\mathbf{u}\}=\{\mathbf{f}\}
$$

where $[\mathbf{K}]$ is the stiffness matrix, and $\{\mathbf{f}\}$ the vector of nodal forces. When the displacement vector $\{\mathbf{u}\}$ is prescribed and if the (unknown) stiffness matrix is not the true one, force residuals $\left\{\mathbf{f}_{r}\right\}$ will arise

$$
\left\{\mathbf{f}_{r}\right\}=[\mathbf{K}]\{\mathbf{u}\}-\{\mathbf{f}\}
$$

Similarly, if the displacement field does not satisfy equilibrium it will induce an equilibrium gap. In the absence of body forces, interior nodes are free from any external load. Consequently, the equilibrium gap method consists in minimizing

$$
\Phi_{m}^{2}=\{\mathbf{u}\}^{t}[\mathbf{K}]^{t}[\mathbf{K}]\{\mathbf{u}\}
$$

where ${ }^{t}$ is the transposition operator, and $\Phi_{m}^{2}$ corresponds to the sum of the squared norm of all equilibrium gaps at interior nodes only. Any displacement field prescribed on the boundary gives rise to a displacement field for which $\Phi_{m}=0$.

This observation requires an additional regularization for boundary nodes. It is proposed to introduce a penalization of short wavelength displacement fluctuations along the edges of the region of interest. The third objective function to be considered has to vanish for any rigid body motion

$$
\Phi_{b}^{2}=\{\mathbf{u}\}^{t}[\mathbf{L}]^{t}[\mathbf{L}]\{\mathbf{u}\}
$$

where $[\mathbf{L}]$ is an operator acting on the ROI boundary [15]. Appendix A details the construction of the new operator proposed herein, which has a simple mechanical interpretation. 


\subsection{Correlation Procedure}

The minimization of correlation residuals $\left(\Phi_{c}^{2}\right)$, equilibrium gap $\left(\Phi_{m}^{2}\right)$, and boundary fluctuations $\left(\Phi_{b}^{2}\right)$, requires the introduction of a total functional $\Phi_{t}$

$$
\left(1+w_{m}+w_{b}\right) \Phi_{t}^{2}=\widetilde{\Phi}_{c}^{2}+w_{m} \widetilde{\Phi}_{m}^{2}+w_{b} \widetilde{\Phi}_{b}^{2}
$$

where $w_{m}$ and $w_{b}$ are weights that define length scales associated with $\widetilde{\Phi}_{m}^{2}$ and $\widetilde{\Phi}_{b}^{2}$, and $\widetilde{\Phi}$ denote normalized residuals. To normalize the residuals, let us consider a displacement field in the form of a plane wave $\mathbf{v}(\mathbf{x})=\mathbf{v}_{0} \exp (i \mathbf{k} \cdot \mathbf{x})$, where $\mathbf{v}_{0}$ is the amplitude and $\mathbf{k}$ the wave vector. The normalized residuals then become

$$
\widetilde{\Phi}_{c}^{2}=\frac{\Phi_{c}^{2}}{\{\mathbf{v}\}^{t}[\mathbf{M}]\{\mathbf{v}\}} \quad, \quad \widetilde{\Phi}_{m}^{2}=\frac{\Phi_{m}^{2}}{\{\mathbf{v}\}^{t}[\mathbf{K}]^{t}[\mathbf{K}]\{\mathbf{v}\}} \quad, \quad \widetilde{\Phi}_{b}^{2}=\frac{\Phi_{b}^{2}}{\{\mathbf{v}\}^{t}[\mathbf{L}]^{t}[\mathbf{L}]\{\mathbf{v}\}}
$$

where $\{\mathbf{v}\}$ collects all the nodal displacements associated with displacement field v. The wavelength dependence of $\{\mathbf{v}\}^{t}[\mathbf{K}]^{t}[\mathbf{K}]\{\mathbf{v}\}$ and $\{\mathbf{v}\}^{t}[\mathbf{L}]^{t}[\mathbf{L}]\{\mathbf{v}\}$ is of fourth order whereas $\{\mathbf{v}\}^{t}[\mathbf{M}]\{\mathbf{v}\}$ is wavelength-independent [15], see Figure 1. The equilibrium gap and boundary regularization kernels thus act as fourth-order low-pass filters, damping deviations from mechanical admissibility and edge displacement fluctuations below a given wavelength. Weights $w_{m}$ and $w_{b}$ are chosen as

$$
w_{m}=\left(2 \pi|\mathbf{k}| \ell_{m}\right)^{4} \quad, \quad w_{b}=\left(2 \pi|\mathbf{k}| \ell_{b}\right)^{4}
$$

where $\ell_{m}$ and $\ell_{b}$ denote the regularization lengths for $\Phi_{m}^{2}$ and $\Phi_{b}^{2}$, respectively. With this choice, $\widetilde{\Phi}_{c}(\mathbf{v})=\widetilde{\Phi}_{m}(\mathbf{v})=\widetilde{\Phi}_{b}(\mathbf{v})=1$ and $\Phi_{t}(\mathbf{v})=1$ if $\ell_{m}=\ell_{b}=$ $1 / 2 \pi|\mathbf{k}|$ 
Figure 1 about here

It is to be emphasized that other forms of regularization kernels could have been chosen. The specific form of the equilibrium gap comes from the strong penalization of high frequency oscillations (fourth power of the wavenumber), and thus also very weak influence on long wavelength modes.

The higher $\ell_{m}$, the more weight is put on the equilibrium gap functional $\widetilde{\Phi}_{m}^{2}$, therefore the equilibrium residuals have to reach lower levels. Similarly, the edge regularization term is designed to have the same scaling properties as the bulk term. The higher $\ell_{b}$, the more weight is put on the boundary functional $\widetilde{\Phi}_{b}^{2}$, therefore the corresponding displacement fluctuations will decrease.

When the material parameters are known, the minimization of $\Phi_{t}^{2}$ with respect to the unknown degrees of freedom can be performed [15]. The above mechanical regularization procedure can be used for an arbitrary supporting mesh, even down to the pixel [14] or voxel [15, 16] scales. In the following, the elements will be considered to be 3-noded triangles (T3) with a linear displacement interpolation.

\subsection{Implementation}

A Newton iterative procedure is followed to circumvent the nonlinear aspect of the minimization problem (i.e., $\Phi_{c}^{2}$ is a nonlinear function of the degrees of freedom). Let $\mathbf{u}^{j}$ denote the displacement field at iteration $j$, and $\{\mathbf{u}\}^{j}$ the vector collecting all the unknown degrees of freedom. By assuming small increments $\mathrm{d} \mathbf{u}=\mathbf{u}^{j+1}-\mathbf{u}^{j}$ of the solution, $\partial \Phi_{t}^{2} / \partial\{\mathbf{u}\}^{j}=\{\mathbf{0}\}$ is recast in a 
matrix-vector product as

$$
([\mathbf{M}]+[\mathbf{N}])\{\mathrm{d} \mathbf{u}\}=\{\mathbf{b}\}^{j}-[\mathbf{N}]\{\mathbf{u}\}^{j}
$$

with

$$
[\mathbf{N}]=w_{m} \frac{\{\mathbf{v}\}^{t}[\mathbf{M}]\{\mathbf{v}\}}{\{\mathbf{v}\}^{t}[\mathbf{K}]^{t}[\mathbf{K}]\{\mathbf{v}\}}[\mathbf{K}]^{t}[\mathbf{K}]+w_{b} \frac{\{\mathbf{v}\}^{t}[\mathbf{M}]\{\mathbf{v}\}}{\{\mathbf{v}\}^{t}[\mathbf{L}]^{t}[\mathbf{L}]\{\mathbf{v}\}}[\mathbf{L}]^{t}[\mathbf{L}]
$$

where $\{\mathbf{b}\}^{j}$ is updated as the picture in the deformed configuration $g$ is corrected by using the current estimate of the displacement field $\mathbf{u}^{j}$. In the present case a cubic gray level interpolation is considered. Conversely, matrices $[\mathbf{M}]$ and $[\mathbf{N}]$ are computed once for all. The iterations stop when the displacement corrections $\{\mathrm{d} \mathbf{u}\}$ reach a small level that is chosen by the user.

In the absence of regularization, the presence of noise leads to secondary minima trapping when small elements are chosen. This is responsible for a degradation of the uncertainty for fine meshes. This secondary minima trapping leads to a multiplicity of stationary solutions of the displacement field. Different solutions are obtained depending on the initial displacement field. Regularization is introduced to mend this problem, at the expense of a priori assumptions on the elastic properties of the considered body. Thus, in the analysis of resolution, a specific pathway consists in first solving the problem with large regularization lengths, and progressively decreasing the regularization length scales $\ell_{m}$ and $\ell_{b}$. Although ending with very low weights given to the regularization, the sought displacement field is driven to a solution where initialization is an "educated guess" based on an elastic interpolation. The final solution will live in an energy landscape where many 
local minima exist, but the selection of the actual minimum results from a convergence history that is expected to be beneficial.

In the following, series of calculations are generally run with different regularization lengths $\ell_{m}$ when $\ell_{b}=\ell_{m} / 2$. Different initial values of $\ell_{m}$ are considered and each time a first calculation is run with an initial value of $\mathbf{u}^{0}=$ 0. The next calculation is carried out with the regularization length divided by two. It takes as initial guess $\mathbf{u}^{0}$ the displacement field at convergence of the previous calculation, and so on. This procedure will be referred to as relaxation. Last, the unstructured mesh is composed of triangles whose typical edge is 10-pixel long.

\section{Artificial Test Cases}

In these first two test cases, a true texture is considered (Figure 2). The picture definition is $531 \times 531$ pixels with an 8-bit digitization. It corresponds to the central part of a cross-shaped sample analyzed in Ref. [20]. To create the deformed configuration, two different displacement fields are considered, namely, a first one that is a sine wave, and a second one that is mechanically admissible. In both cases, a linear interpolation of the gray levels is used to generate picture $g$ in the deformed configuration. No noise is included. The same mesh is used in both cases and is made of 2313 inner nodes and 197 edge nodes.

Figure 2 about here 


\subsection{Sine Wave}

The sine wave is an interesting case since it corresponds to one of the standard baseline analyses for optical systems (in particular in the context of DIC [3]). The choice of the spatial resolution has to be compared with the wave length of the prescribed displacement. The two components of the latter read

$$
u_{x}(x, y)=2 \sin \left(2 \pi x / n_{x}\right) \quad, \quad u_{y}(x, y)=2 \sin \left(2 \pi y / n_{y}\right)
$$

where $n_{x} \times n_{y}$ is the number of pixels in the ROI.

It is worth noting that the normalized correlation residual $\widetilde{\Phi}_{c}^{2}$ was introduced to evaluate the change in the correlation functional for a perturbation in the displacement field, and to compare it to a similar perturbation in the regularization functional. The value of the absolute minimum of $\Phi_{c}$ is irrelevant. Thus only the parabolic expansion of the functional $\Phi_{c}^{2}$ was considered. However, to judge the quality of the image registration itself, it is more relevant to evaluate the norm of $\varphi_{c}$-field. The latter, however can be affected by a mean change of gray levels between the pictures in the deformed and reference configurations. To cancel out this effect (simply contributing as an offset in gray levels), it is convenient to resort to the standard deviation of $\varphi$. A dimensionless estimator consists in comparing this standard deviation to the dynamic range $\Delta f=\max (f)-\min (f)$ of the reference picture. Thus $\hat{\Phi}_{c}^{2}=\left[\left\langle\varphi^{2}\right\rangle-\langle\varphi\rangle^{2}\right] /(\Delta f)^{2}$, where $\langle\bullet\rangle$ denotes an average of the ROI. $\hat{\Phi}_{c}$ will be used in the sequel for estimating the quality of image registration.

Figure 3 shows the change of the standard error between the measured and prescribed displacement field for the different regularization lengths $\ell_{m}$ when $\ell_{b} / \ell_{m}$ is set to different values. The same trends are observed for the 
two components of the displacement field. Consequently, only one result is reported in Figure 3. When the edge regularization is too large (i.e., $\ell_{b} / \ell_{m}>$ 1), the error is high. This corresponds to an interpolation error since the displacement fluctuations are filtered out. As the edge regularization length decreases, the error decreases too. In the present case, $\ell_{b} / \ell_{m}$ ratios less than 1 lead to very good results even if $\ell_{m}$ is relaxed to very small values. This observation justifies the choice of selecting a fixed ratio $\ell_{b} / \ell_{m}=1 / 2$ and decreasing both lengths at the same time.

Figure 3 about here

All these trends are confirmed when the mean correlation residuals are analyzed in Figure 4. For a large range of regularization length ratios, the levels of correlation residuals are virtually identical. The image registration is of comparable quality. The only difference is given by the weight put on the mechanical and edge regularizations. This choice is left to the user and its understanding of the underlying behavior of the studied material. The same can be said for the edge regularization, which is related to the boundary conditions of the mechanical analysis when DIC is assisted by mechanics.

Figure 4 about here

Figure 5 shows the change of the standard deviation between the measured and prescribed displacement fields for the different cases when $\ell_{b} / \ell_{m}=$ $1 / 2$. The standard displacement error is virtually identical for the two displacement components (except for very large initial regularization lengths), even though an unstructured mesh is used. No bias is noted due to the fact 
that the mesh is not regular. Different trends are observed. First for very large initial regularization lengths (i.e., $\ell_{m}>n_{x}, n_{y}$ ), the standard error is very high. This is caused by interpolation errors as very large regularization lengths prevent from assessing small displacement fluctuations. When they are relaxed, a smaller but still significant error is observed. Conversely, for small regularization lengths, the standard error remains small but the relaxation increases slightly the error. When an initial value $\ell_{m}=128$ pixels is chosen, the smallest errors are observed. For initial lengths less than $\ell_{m}=128$ pixels, error levels of the order of or less than $10^{-2}$ pixel are achieved.

\section{Figure 5 about here}

For the same series of computations, Figure 6(a) shows the change of the dimensionless correlation residuals $\hat{\Phi}_{c}$. When the initial regularization lengths are such that $\ell_{m}<n_{x}, n_{y}$, the correlation residuals reach very similar values, thereby indicating that the results are trustworthy. For the quality of image registration, it indicates that initial regularization lengths $\ell_{m}$ less than 256 pixels, the overall registration quality is very close for any initial or subsequent regularization length. This conclusion is in very good agreement with what is observed in Figure 5. For large initial regularization lengths, the mean correlation residuals reach high levels (i.e., almost an order of magnitude higher). This is the consequence of the interpolation error discussed before, as the sought displacement field does not belong to the kernel of the regularization operator. The next choice is then to seek more or less mechanical content in the measured displacement field. This is characterized by 
the equilibrium gap whose mean normalized value is shown in Figure 6(b). For larger regularization lengths, the measured field displays less equilibrium gap as its weight in the total functional (10) is increased. There is a whole range of initial values $\left(128<\ell_{m}<512\right.$ pixels $)$ for which the equilibrium gap is independent of the initial value of $\ell_{m}$. This zone is the most favorable in the present case.

Figure 6 about here

In all the following analyses, the ratio $\ell_{b} / \ell_{m}$ is set to $1 / 2$. It follows that there is more weight put to the mechanical regularization than the edge regularization. The former will be mainly studied in the remainder of the paper.

\subsection{Mechanics-Based Displacement}

An elastic calculation (with Poisson's ratio $\nu=0.3$ ) is performed on a plate whose size is exactly the same as the reference picture shown in Figure 2. The boundary of the top part only moves in the horizontal direction, and the boundary of the bottom part has a prescribed displacement that is again a sine wave of unitary amplitude and wave length equal to the length of the lower edge. A mesh consisting of $50 \times 50$ Q4 elements is considered. The corresponding displacement is subsequently interpolated at the pixel level by using bilinear shape functions of Q4 elements. Figure 7 shows the displacement components that are used to deform the reference picture of Figure 2. The range of vertical displacement is equal to 2 pixels, and that of the horizontal displacements is of the order of 1.7 pixel. 
Figure 7 about here

The same type of computation as before is performed in the present case when $\ell_{b} / \ell_{m}=1 / 2$. Figure 8 shows the change of the standard displacement. The same trends are observed when compared to Figure 5 for the same reasons. For initial regularization lengths $\ell_{m}$ ranging from 64 to 512 pixels the error level is less than $10^{-2}$ pixel at the end of the relaxation process. It is worth remembering that this error includes the approximations of the reference solution (on a regular Q4 mesh), those of the T3 mesh used in the DIC analysis, and the inevitable gray level interpolation.

Figure 8 about here

The same conclusions can be drawn when the trends observed in terms of correlation residuals (Figure 9(a)) and equilibrium residuals (Figure 9(b)) are compared with those of the previous test case.

Figure 9 about here

There are however differences to be noted. First, the correlation residuals do not vary over a large dynamic range when compared to the previous case. It is concluded that even though large regularization lengths are used, they still allow for more fluctuations of the displacement field. Second, the minimum levels of the equilibrium residuals are less than those observed in the previous case. This is to be expected since the present test case is associated with a mechanically admissible displacement field (in an FE sense). 
From the results of the two test cases discussed in this section, it is concluded that the implementation of mechanics-aided DIC (or MA-DIC) is validated. The next section will deal with image pairs obtained during an experimental test.

\section{Analysis of Two Different Textures}

In the following analyses, experimental data are considered. A cross-shaped sample made of nodular graphite cast iron was loaded equibiaxially along two perpendicular directions (i.e., $F_{1}=F_{2}=F$, where $F_{1}$ and $F_{2}$ are the applied forces along the two directions). One surface of the sample was observed at the macroscale (picture definition: $1024 \times 1024$ pixels, 12-bit digitization, telecentric lens), and the other one at the mesoscale (picture definition: $1024 \times 1280$ pixels, 12-bit digitization, telecentric lens), see Figure 10 . The observation of the former required to spray black and white paint to enable DIC analyses. The latter is a natural texture, which is very difficult to analyze with conventional DIC procedures. The physical size of one pixel is $6.7 \mu \mathrm{m}$ for the mesoscale picture and $48 \mu \mathrm{m}$ for the macroscale picture.

\section{Figure 10 about here}

\subsection{Natural Texture}

The image pair considered in this section corresponds to two consecutive pictures shot when the sample was already mounted in the testing machine with $F=0$. This analysis is performed to evaluate the resolution of the 
measurement technique [1]. Figure 11 shows the standard displacement resolution for the two displacement components of displacement. The larger the regularization length, the smaller the displacement resolution. It is worth noting that any value less than one centipixel is very seldom encountered. This is even more noteworthy that the texture of the pictures is very difficult to analyze. To get all the results reported in Figure 11 (requiring 51 simulations), less than one hour of CPU time was needed on an Intel Xeon X5660 processor. The mesh consisted of 8,749 nodes, and therefore involved 17,498 kinematic unknowns.

To compare the present results with more standard approaches, a local and global Q4-DIC analysis is run. It is based on 4-noded ZOIs for the local approach, and 4-noded elements in a global approach. In both cases a bilinear displacement is considered. The implementation details of both approaches follow very closely those used herein [12] except that no regularization length is used. The right envelope of the regularized results virtually coincides with those of the global Q4-DIC analysis. The global analysis, as expected when the same ZOI and element size is considered, out-performs the local analysis [12]. With this difficult texture, there is a clear gain to consider global vs. local approaches. The regularization proposed herein induces a very significant additional gain as all the non-mechanical fluctuations are filtered out. Last, the relaxation process of the regularization length leads to very significant reductions of the displacement fluctuations.

Figure 11 about here

Figure 12(a) shows the dimensionless correlation residuals for different 
initial regularization lengths. Their level remains almost constant and very small for any value of the correlation length. This result indicates that in the present case, it is impossible to discriminate any displacement from the analysis of the correlation residuals alone. However, the equilibrium residuals (Figure 12(b)) vary very strongly with the regularization lengths. In this particular case, the gain provided by the mechanical regularization is spectacular, namely, all the spurious fluctuations are filtered out.

Figure 12 about here

If an estimate of the displacement field is sought with a very light mechanical regularization, the procedure to follow is to run a first correlation with a very large regularization length, say $\ell_{m}=1024$ pixels. At convergence, a second calculation could be run $\ell_{m}=512$ pixels by using an initial guess that is the displacement field at convergence with $\ell_{m}=1024$ pixels. If this path is followed, it can be stopped when $\ell_{m}=1$ pixel. The result given in Figure 11 shows that the displacement resolution is very small and the equilibrium residual remains very small (Figure 12(b)).

The next issue is related to the choice of the variation of the regularization length between two consecutive computations. Figure 13 shows the standard displacement resolution for the two components of displacement when different ratios are applied to the regularization lengths. If a very small regularization is considered and initialized with the result obtained for $\ell_{m}=1024$ pixels, the displacement resolution remains less than when the previous relaxation process is followed. With this route, the displacement resolution becomes even smaller and virtually independent of the final 
regularization length.

\section{Figure 13 about here}

Figure 14(a) shows that the dimensionless correlation residuals are constant for all the computations with this procedure. The equilibrium residuals (Figure 14(b)) vary but not strongly when the regularization length is reduced. This result shows that there is no need to run numerous calculations to relax the regularization. Only a first computation is needed with a large regularization length, say $\ell_{m}=1024$ pixels, followed by a second one for a small length (i.e., values as low as $\ell_{m}=1$ pixel can be considered).

\section{Figure 14 about here}

\subsection{Artificial Texture}

The image pair correlated hereafter corresponds to a reference configuration when $F=0$, and a deformed configuration for which $F=25 \mathrm{kN}$. When this load level is reached, the entire sample still lies within the elastic domain. In the present case, the boundary of the ROI is adapted to the geometry of the sample (Figure 15). No special care was exercised to match the connecting radii to the straight parts. Consequently, the edge regularization discussed previously is still used. Had the geometry been fully match with the external boundary, a traction-free condition could have been used instead.

Figure 15 about here

Figure 16(a) shows the dimensionless correlation residuals for different initial regularization lengths with the same type of computations as before 
$\left(\ell_{b} / \ell_{m}=1 / 2\right)$. Their level remains almost constant for any value of the correlation length and very low. This result indicates that all the evaluations are close. However, the equilibrium residuals (Figure 16(b)) are the smallest for large initial regularization lengths.

Figure 16 about here

For small initial regularization lengths, the equilibrium residuals are less than those observed in the previous case. This result allows us to fully relax the regularization length (i.e., $\ell_{m}=1$ pixel) and even start with a modest one (e.g., $\ell_{m}=64$ or 32 pixels). This is made possible thanks to the texture quality that is better than that of the previous approach.

Figure 17 shows the displacement components that are measured when $\ell_{m}=1$ pixel with an initial value of 32,128 and 1024 pixels. The range of vertical displacement is equal to 0.9 pixel, and that of the horizontal displacements is about 1 pixel. There is a clear strain concentration in the central part of the sample, where the transverse thickness is reduced (Figure 10(a)). Note that this effect is not taken into account in the regularization kernel where plane elasticity is assumed to hold. As the initial regularization length increases, the displacement fluctuations of the final results decrease because the relaxation process does not lead to the same solution.

Figure 17 about here 


\section{$5 \quad$ Summary and Perspectives}

Introducing a mechanically-based regularization in a finite-element formulation of DIC was shown to lead to a significant reduction of uncertainty levels for artificial non-uniform displacement fields. Application of the same methodology to a real experimental case showed that even poor image textures could be dealt with successfully. In the present analyses, discretizations based upon unstructured meshes made of 3-noded triangles (i.e., T3-DIC) were considered in the computations. The latter are less demanding than with a pixel-scale DIC approach. However, it is believed that the results presented herein are generic and should apply to pixel-scale DIC procedures.

The fact that a regularization could reduce the uncertainty in the same way as coarsening a spatial discretization has been reported in the past [15]. The novelty of the procedure proposed herein is that the resolution / regularization length limit can be broken through an appropriate driving of the regularized DIC algorithm. The mechanical kernel introduced in the global functional may not provide a genuine picture of the actual displacement field (see the sine wave displacement field as an artificial case study, or the last example of a specimen with a varying thickness). Thus rather than prescribing a large regularization length scale, the latter can be considered in a transient stage of the algorithm to help dealing with large displacements, and / or poor textures (see the picture of SG cast iron at the mesoscale). This regularization provides only an "educated guess" for the displacement

field, where the actual freedom of DIC is very much constrained. However, at convergence, relaxing the regularization length scale to very small val- 
ues (and hence playing only a negligible role) allows the DIC procedure to inherit from a displacement field that is much closer to the actual displacement field. Hence, spurious pinning of the estimated displacement field in local secondary minima is drastically reduced. However, this reduction is not complete, and if the initial regularization length scale is too small, some amount of random pinning is still present at the initial stage, and cannot be relaxed upon further relaxation steps. Figure 17 is an illustration of this property. The existence of history-dependent solutions (i.e., not controlled exclusively by the value of the element size $\ell$ ) is a clear sign of multiple minima, calling for adapted strategies to avoid (or limit) spurious trapping.

The philosophy of this procedure can be compared with the pyramidal multiscale approach proposed in [11]. There again, kinematic degrees of freedom are reduced and local minima avoided in transient stages of the algorithm. At each scale, the converged field is used as an initialization for the finer scale and hence this multiscale driving provided both robustness and lower uncertainties. However, such a multiscale procedure is not easily implemented with an unstructured mesh decomposition, unless the mesh is endowed with a hierarchical structure. In contrast, the mechanical regularization easily complies with an arbitrary discretization.

\section{Acknowledgments}

This work was supported by a grant from Région Île-de-France (plateforme francilienne d'expérimentation mécanique de troisième génération). ZT thanks the International Affairs Bureau of École Normale Supérieure 
de Cachan for its support during his stay at LMT-Cachan.

\section{References}

[1] ISO/IEC guide 99-12:2007. International Vocabulary of Metrology - Basic and General Concepts and Associated Terms, VIM. International Organization for Standardization: Geneva (Switzerland); 2007.

[2] Bergonnier S, Hild F, Roux S. Digital image correlation used for mechanical tests on crimped glass wool samples. J. Strain Analysis 2005; 40(2):185-197.

[3] Bornert M, Brémand F, Doumalin P, Dupré J-C, Fazzini M, Grédiac M, Hild F, Mistou S, Molimard J, Orteu J-J, Robert L, Surrel Y, Vacher P, Wattrisse B. Assessment of Digital Image Correlation measurement errors: Methodology and results. Exp. Mech. 2009; 49(3):353-370.

[4] Lucas BD, Kanade T. An Iterative Image Registration Technique with an Application to Stereo Vision. Proceedings 1981 DARPA Imaging Understanding Workshop. 1981; 121-130.

[5] Burt PJ, Yen C, Xu X. Local correlation measures for motion analysis: a comparative study. Proceedings IEEE Conf. on Pattern Recognition and Image Processing. 1982; 269-274.

[6] Sutton MA, Wolters WJ, Peters WH, Ranson WF, McNeill SR. Determination of Displacements Using an Improved Digital Correlation Method. Im. Vis. Comp. 1983; 1(3):133-139. 
[7] Sutton MA, McNeill SR, Helm JD, Chao YJ. Advances in TwoDimensional and Three-Dimensional Computer Vision. Photomechanics, Rastogi PK, ed. Springer: Berlin (Germany), 2000; 323-372.

[8] Sutton MA, Orteu J-J, Schreier H. Image correlation for shape, motion and deformation measurements: Basic Concepts, Theory and Applications. Springer: New York, NY (USA); 2009.

[9] Broggiato GB. Adaptive image correlation technique for full-field strain measurement. Proceedings 12th Int. Conf. Exp. Mech. 2004; 420-421.

[10] Sun Y, Pang J, Wong C, Su F. Finite-element formulation for a digital image correlation method. Appl. Optics 2005; 44(34):7357-7363.

[11] Besnard G, Hild F, Roux S. "Finite-element" displacement fields analysis from digital images: Application to Portevin-Le Chatelier bands. Exp. Mech. 2006; 46:789-803.

[12] Hild F, Roux S. Comparison of local and global approaches to digital image correlation. Exp. Mech. 2012; in press, DOI 10.1007/s11340-012$9603-7$.

[13] Rupil J, Vincent L, Hild F, Roux S. Probabilistic modeling of mesocrack initiations in 304L stainless steel. Int. J. Multiscale Comput. Eng. 2011; $9(4): 445-458$.

[14] Passieux J-C, Périé J-N. Digital image correlation using proper generalized decomposition: PGD-DIC. Int. J. Num. Meth. Eng. 2012; submitted. 
[15] Leclerc H, Périé J-N, Roux S, Hild F. Voxel-scale digital volume correlation. Exp. Mech. 2011; 51(4):479-490.

[16] Leclerc H, Périé J-N, Hild F, Roux S. Digital Volume Correlation: What are the limits to the spatial resolution? Mech. E Ind. 2012; in press, DOI: $10.1051 / \mathrm{meca} / 2012025$.

[17] Réthoré J, Roux S, Hild F. An extended and integrated digital image correlation technique applied to the analysis fractured samples. Eur. J. Comput. Mech. 2009; 18:285-306.

[18] Tikhonov AN, Arsenin VY. Solutions of ill-posed problems. J. Wiley: New York (USA); 1977.

[19] Bonnet M, Constantinescu A. Inverse problems in elasticity. Inverse Problems 2005; 21:R1-R50.

[20] Claire D, Hild F, Roux S. A finite element formulation to identify damage fields: The equilibrium gap method. Int. J. Num. Meth. Engng. 2004; 61(2):189-208.

[21] Fedele R, Raka B, Hild F, Roux S. Identification of adhesive properties in GLARE assemblies by Digital Image Correlation. J. Mech. Phys. Solids $2009 ; 57: 1003-1016$.

[22] Wei Z, Deng X, Sutton MA, Yan J, Cheng C-S, Zavattieri P. Modeling of mixed-mode crack growth in ductile thin sheets under combined in-plane and out-of-plane loading. Eng. Fract. Mech. 2011; 78:3082-3102. 
[23] Leclerc H, Périé J-N, Roux S, Hild F. Integrated digital image correlation for the identification of mechanical properties. MIRAGE 2009, Gagalowicz A, Philips W, eds. Springer: Berlin, 2009; 161-171. 


\section{Appendix A: Edge regularization}

In this appendix, the edge regularization is presented. To be consistent with the bulk regularization, a local quadratic form is sought on the nodal displacement that has to be invariant under a rigid body motion. The consequence of this requirement is that such a quadratic form will have a simple mechanical interpretation. Local in the context of a discretized medium means that the edge regularization will only be based on nearest neighbors. Thus, focusing on specific node, $M_{n}$, only the three consecutive nodes located at points ( $\left.M_{n-1}, M_{n}, M_{n+1}\right)$ will be considered (irrespective of their alignment or not). Because of translational invariance, one may choose $M_{n}$ as the origin, so that the geometry is captured through the two vectors $\boldsymbol{\zeta}_{-1}=\mathbf{M}_{\mathbf{n}} \mathbf{M}_{\mathbf{n}-\mathbf{1}}$ and $\boldsymbol{\zeta}_{1}=\mathbf{M}_{\mathbf{n}} \mathbf{M}_{\mathbf{n}+\mathbf{1}}$. It is convenient to introduce the orthogonal vectors of $\boldsymbol{\zeta}_{-1}$ and $\boldsymbol{\zeta}_{1}$ rotated by $\pi / 2$, resp. $\boldsymbol{\tau}_{-1}$ and $\boldsymbol{\tau}_{1}$.

The displacement vector at those nodes is denoted by $\left(\mathbf{u}_{n-1}, \mathbf{u}_{n}, \mathbf{u}_{n+1}\right)$. Invariance under translation imposes that the regularization functional should be a quadratic form of $\left(\mathbf{v}_{-1}, \mathbf{v}_{1}\right)=\left(\mathbf{u}_{n-1}-\mathbf{u}_{n}, \mathbf{u}_{n+1}-\mathbf{u}_{n}\right)$. Invariance under rotation imposes that the functional should be invariant under the following change in the displacement field, where $\boldsymbol{\omega}$ and $\omega$ denotes respectively a rotation vector, and its magnitude.

$$
\begin{aligned}
& \mathbf{v}_{-1}=\boldsymbol{\omega} \times \boldsymbol{\zeta}_{-1}=\omega \boldsymbol{\tau}_{-1} \\
& \mathbf{v}_{1}=\boldsymbol{\omega} \times \boldsymbol{\zeta}_{1}=\omega \boldsymbol{\tau}_{1}
\end{aligned}
$$

Three scalars $a_{n}(n=1,2,3)$ can be formed, which are invariant under such a rotation. The first two depend on a pair of neighboring node displacement, 
namely,

$$
\begin{aligned}
& a_{1}=\frac{\boldsymbol{\zeta}_{-1} \cdot \mathbf{v}_{-1}}{\left\|\boldsymbol{\zeta}_{-1}\right\|^{2}} \\
& a_{2}=\frac{\boldsymbol{\zeta}_{1} \cdot \mathbf{v}_{1}}{\left\|\boldsymbol{\zeta}_{1}\right\|^{2}}
\end{aligned}
$$

where the normalization has been introduced here for reasons that will become clearer later. The third scalar reads

$$
a_{3}=\frac{\boldsymbol{\tau}_{1} \cdot \mathbf{v}_{1}}{\left\|\boldsymbol{\tau}_{1}\right\|^{2}}-\frac{\boldsymbol{\tau}_{-1} \cdot \mathbf{v}_{-1}}{\left\|\boldsymbol{\tau}_{-1}\right\|^{2}}
$$

Thus the most general form for the sought functional is expressed as

$$
\mathcal{T}_{b}=\frac{1}{2} \sum_{n} \alpha_{n} a_{n}^{2}
$$

where $\alpha_{n}$ are arbitrary factors. The physical interpretation of those terms is straightforward. The first two correspond to a linear spring connecting nodes $\left(M_{n-1}, M_{n}\right)$ and $\left(M_{n}, M_{n+1}\right)$, respectively. Parameters $\alpha_{1}$ and $\alpha_{2}$ are the stiffness of these springs. The last invariant, $a_{3}$, corresponds to the resulting moment of forces at node $M_{n}$, and hence the last term in the functional would correspond to an angular spring. In the absence of additional information, it is natural to choose all stiffnesses as constant, say equal to unity, and the total magnitude of the functional being set with respect to the other functionals as discussed in the main text.

To make the expression of this functional more explicit, the 'rigidity' matrix $[\mathbf{L}]$ introduced in the main text is obtained by assembling (with respect to all boundary nodes $i$ ) the local contributions

$$
\mathcal{T}_{b}=\frac{1}{2}\left(\begin{array}{ll}
\mathbf{v}_{1} & \mathbf{v}_{-1}
\end{array}\right) \cdot\left(\begin{array}{cc}
\frac{\boldsymbol{\zeta}_{1} \otimes \boldsymbol{\zeta}_{1}}{\left\|\boldsymbol{\zeta}_{1}\right\|^{4}}+\frac{\boldsymbol{\tau}_{1} \otimes \boldsymbol{\tau}_{1}}{\left\|\boldsymbol{\tau}_{1}\right\|^{4}} & -\frac{\boldsymbol{\tau}_{1} \otimes \boldsymbol{\tau}_{-1}}{\left\|\boldsymbol{\tau}_{1}\right\|^{2}\left\|\boldsymbol{\tau}_{-1}\right\|^{2}} \\
-\frac{\boldsymbol{\tau}_{-1} \otimes \boldsymbol{\tau}_{1}}{\left\|\boldsymbol{\tau}_{1}\right\|^{2}\left\|\boldsymbol{\tau}_{-1}\right\|^{2}} & \frac{\boldsymbol{\zeta}_{-1} \otimes \boldsymbol{\zeta}_{-1}}{\left\|\boldsymbol{\zeta}_{-1}\right\|^{4}}+\frac{\boldsymbol{\tau}_{-1} \otimes \boldsymbol{\tau}_{-1}}{\left\|\boldsymbol{\tau}_{-1}\right\|^{4}}
\end{array}\right) \cdot\left(\begin{array}{c}
\mathbf{v}_{1} \\
\mathbf{v}_{-1}
\end{array}\right)
$$


Noting that $\left\|\boldsymbol{\tau}_{n}\right\|=\left\|\boldsymbol{\zeta}_{n}\right\|$, and $\boldsymbol{\zeta}_{n} \otimes \boldsymbol{\zeta}_{n}+\boldsymbol{\tau}_{n} \otimes \boldsymbol{\tau}_{n}=\left\|\boldsymbol{\zeta}_{n}\right\|^{2} \mathbf{I}$, the particular choice of 'elastic' constants, $\alpha_{n}$, leads to the simplified expression

$$
\mathcal{T}_{b}=\frac{1}{2\left\|\boldsymbol{\zeta}_{1}\right\|^{2}\left\|\boldsymbol{\zeta}_{-1}\right\|^{2}}\left(\begin{array}{cc}
\mathbf{v}_{1} & \mathbf{v}_{-1}
\end{array}\right) \cdot\left(\begin{array}{cc}
\left\|\boldsymbol{\zeta}_{-1}\right\|^{2} \mathbf{I} & -\boldsymbol{\tau}_{1} \otimes \boldsymbol{\tau}_{-1} \\
-\boldsymbol{\tau}_{-1} \otimes \boldsymbol{\tau}_{1} & \left\|\boldsymbol{\zeta}_{1}\right\|^{2} \mathbf{I}
\end{array}\right) \cdot\left(\begin{array}{c}
\mathbf{v}_{1} \\
\mathbf{v}_{-1}
\end{array}\right)
$$

To conclude the final expression of the edge regularization matrix, the expression of $\mathbf{v}$ as a function of the nodal displacement is to be used to convert the above quadratic form into

$$
\mathcal{T}_{b}=\left(\begin{array}{lll}
\mathbf{u}_{i+1} & \mathbf{u}_{i} & \mathbf{u}_{i-1}
\end{array}\right) \cdot[\mathbf{L}] \cdot\left(\begin{array}{c}
\mathbf{u}_{i+1} \\
\mathbf{u}_{i} \\
\mathbf{u}_{i-1}
\end{array}\right)
$$

with $[\mathbf{L}]=\frac{1}{2\left\|\boldsymbol{\zeta}_{1}\right\|^{2}\left\|\boldsymbol{\zeta}_{-1}\right\|^{2}}[\boldsymbol{\ell}]$

$$
\begin{aligned}
& \ell_{11}=\left\|\boldsymbol{\zeta}_{-1}\right\|^{2} \mathbf{I} \\
& \ell_{12}=-\left\|\boldsymbol{\zeta}_{-1}\right\|^{2} \mathbf{I}+\boldsymbol{\tau}_{1} \otimes \boldsymbol{\tau}_{-1} \\
& \ell_{13}=-\boldsymbol{\tau}_{1} \otimes \boldsymbol{\tau}_{-1} \\
& \ell_{21}=-\left\|\boldsymbol{\zeta}_{-1}\right\|^{2} \mathbf{I}+\boldsymbol{\tau}_{-1} \otimes \boldsymbol{\tau}_{1} \\
& \ell_{22}=\left(\left\|\boldsymbol{\zeta}_{-1}\right\|^{2}+\left\|\boldsymbol{\zeta}_{1}\right\|^{2}\right) \mathbf{I}-\boldsymbol{\tau}_{1} \otimes \boldsymbol{\tau}_{-1}-\boldsymbol{\tau}_{-1} \otimes \boldsymbol{\tau}_{1} \\
& \ell_{23}=-\left\|\boldsymbol{\zeta}_{1}\right\|^{2} \mathbf{I}+\boldsymbol{\tau}_{1} \otimes \boldsymbol{\tau}_{-1} \\
& \ell_{31}=-\boldsymbol{\tau}_{-1} \otimes \boldsymbol{\tau}_{1} \\
& \ell_{32}=\left\|\boldsymbol{\zeta}_{1}\right\|^{2} \mathbf{I}+\boldsymbol{\tau}_{-1} \otimes \boldsymbol{\tau}_{1} \\
& \ell_{33}=\left\|\boldsymbol{\zeta}_{1}\right\|^{2} \mathbf{I}
\end{aligned}
$$




\section{Appendix B: Main notations}

$\{$ b $\}$ correlation vector

$\left\{\mathbf{b}^{j}\right\} \quad$ correlation vector at iteration $n$

$f \quad$ picture in the reference configuration

$\{\mathbf{f}\} \quad$ nodal force vector

$\left\{\mathbf{f}_{r}\right\} \quad$ residual force vector

F $\quad$ applied load level

$g \quad$ picture in the deformed configuration

i imaginary unit

j iteration number

$\mathbf{k} \quad$ wave vector

$[\mathbf{K}] \quad$ stiffness matrix

$\ell_{m}, \ell_{b} \quad$ regularization lengths

[L] edge regularization matrix

$[\mathrm{M}] \quad$ correlation matrix

[N] global matrix

$n \quad$ index

$n_{x}, n_{y}$ number of pixels in the ROI

$u_{n} \quad$ degree of freedom

$\mathbf{u} \quad$ displacement vector

$u_{x}, u_{y}$ displacement components

$\mathbf{u}^{0} \quad$ initial displacement vector

du displacement increment vector

$\{\mathbf{u}\} \quad$ vector collecting all kinematic degrees of freedom

$\left\{\mathbf{u}^{j}\right\} \quad$ vector collecting all kinematic degrees of freedom at iteration $n$ 


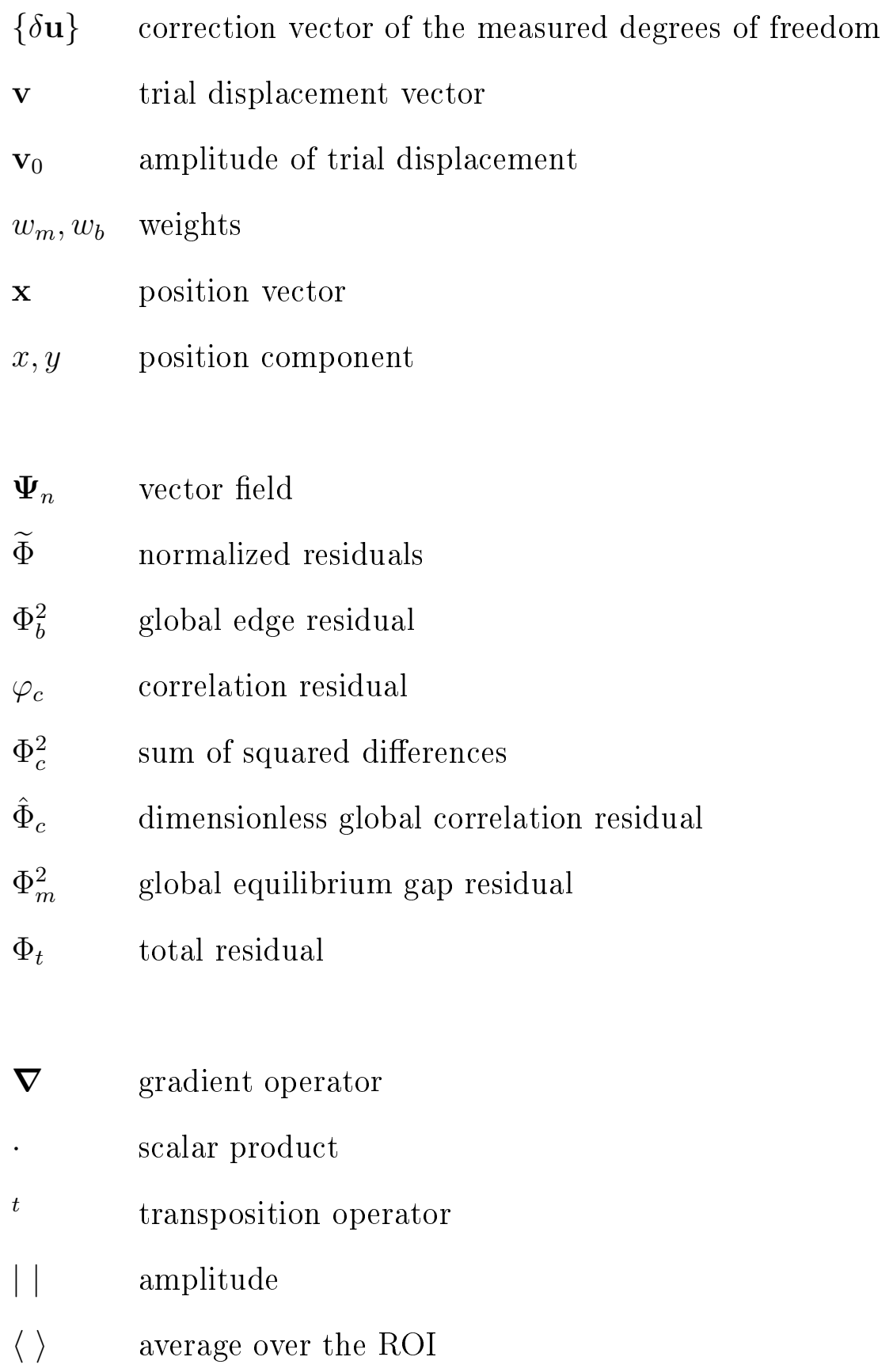




\section{List of Figures}

1 Schematic log-log plot of the dependence of the three functionals and the corresponding normalizing terms with the norm of the wavevector $\mathbf{k}$. The DIC functional (bold solid line) is wavevector-independent. The regularization functionals (dashed lines) are chosen to have a steep slope of 4 , so as to define precisely the filtering frequency. Edge regularization is parallel and chosen to be always lower than the bulk one. . 35

2 Reference picture of the first two test cases. . . . . . . . . . 36

3 Change of the standard displacement error for different regularization lengths and different $\ell_{b} / \ell_{m}$ ratios. For each curve, the relaxation process is to be read from right to left. . . . . 37

4 Change of the dimensionless correlation residual for different regularization lengths and different $\ell_{b} / \ell_{m}$ ratios. For each curve, the relaxation process is to be read from right to left. . 38

5 Change of the standard displacement error for different regularization lengths and different initialization lengths $\ell_{m}$ (in pixels) indicated in the legend. . . . . . . . . . . . . . 39

6 Change of the dimensionless correlation residuals (a) and equilibrium residuals (b) for different regularization lengths and different initialization lengths $\ell_{m}$ (in pixels) indicated in the legend. . . . . . . . . . . . . . . . 4 40 
$7 \quad$ Prescribed displacement field in the mechanical test case in the vertical (a) and horizontal (b) directions. The displacements are expressed in pixels. . . . . . . . . . . . . . . . . . 41

8 Change of the standard displacement error for different regularization lengths and different initialization lengths $\ell_{m}$ (in pixels) indicated in the legend. . . . . . . . . . . . . . . . 42

9 Change of the dimensionless correlation residuals (a) and equilibrium residuals (b) for different regularization lengths and different initialization lengths $\ell_{m}$ (in pixels) indicated in the legend. . . . . . . . . . . . . . . . 43

10 Reference pictures at the macroscopic (a) and mesoscopic (b) scales of a cross-shaped sample made of nodular graphite cast iron. . . . . . . . . . . . . . . . 44

11 Standard displacement resolution in the vertical (a) and horizontal (b) directions for different regularization lengths $\ell_{m}$, ZOI (dashed blue line) or element (solid blue line) sizes $\ell$ when $\ell_{b} / \ell_{m}=1 / 2$. The legend indicates the initial length

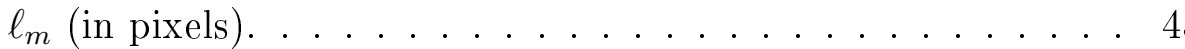

12 Change of the dimensionless correlation residuals (a) and equilibrium residuals (b) for different regularization lengths and different initializations. Note the narrow range of variation of $\hat{\Phi}_{c} \ldots \ldots \ldots \ldots \ldots \ldots \ldots$ 
13 Standard displacement resolution in the vertical (a) and horizontal (b) directions for different regularization lengths and different number of jumps of the regularization length (indicated in the legend). . . . . . . . . . . . . . 47

14 Change of the dimensionless correlation residuals (a) and equilibrium residuals (b) for different regularization lengths and and different number of jumps (indicated in the legend) to decrease the regularization length. Note the narrow range of variation of $\hat{\Phi}_{c} \ldots \ldots \ldots \ldots \ldots$

15 Mesh made of T3 elements used in the MA-DIC analyses at the macroscopic scale (see Figure 10(a)) . . . . . . . . . . 49

16 Change of the dimensionless correlation residuals (a) and equilibrium residuals (b) for different regularization lengths and different initialization lengths $\ell_{m}$ (in pixels) indicated in the legend. . . . . . . . . . . . . . . 50

17 Measured displacement field in the biaxial test in the vertical (left) and horizontal (right) directions. Three initial regularization lengths $\ell_{m}$ are considered. The final regularization length is identical $\left(\ell_{m}=1\right.$ pixel). The displacements are expressed in pixels. . . . . . . . . . . . . . . 51 


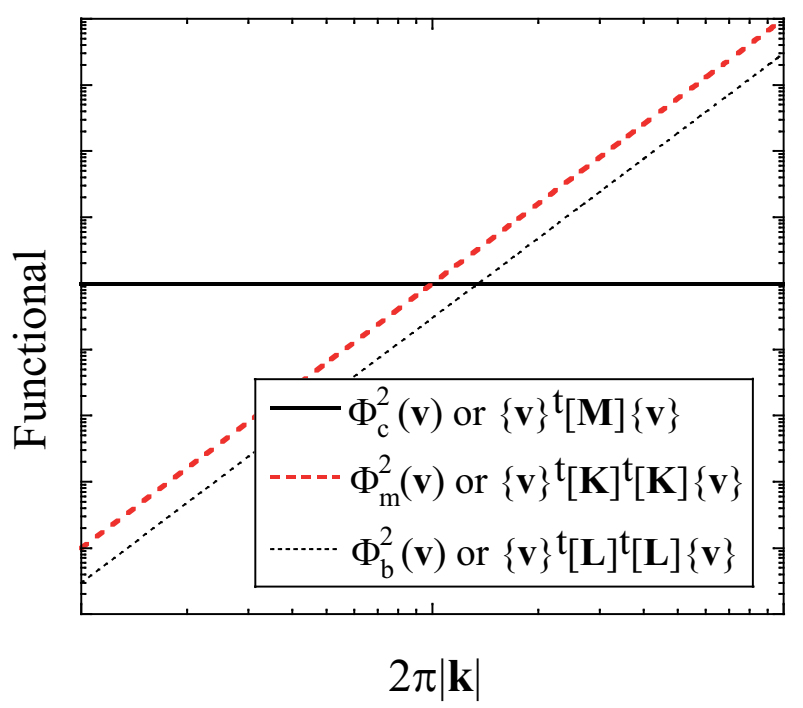

Figure 1: Schematic log-log plot of the dependence of the three functionals and the corresponding normalizing terms with the norm of the wavevector k. The DIC functional (bold solid line) is wavevector-independent. The regularization functionals (dashed lines) are chosen to have a steep slope of 4, so as to define precisely the filtering frequency. Edge regularization is parallel and chosen to be always lower than the bulk one. 


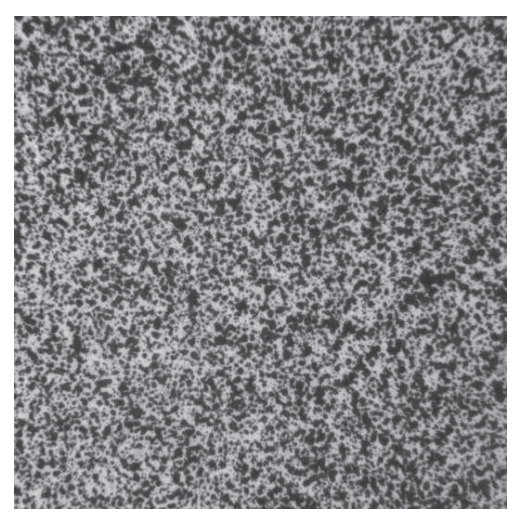

Figure 2: Reference picture of the first two test cases. 


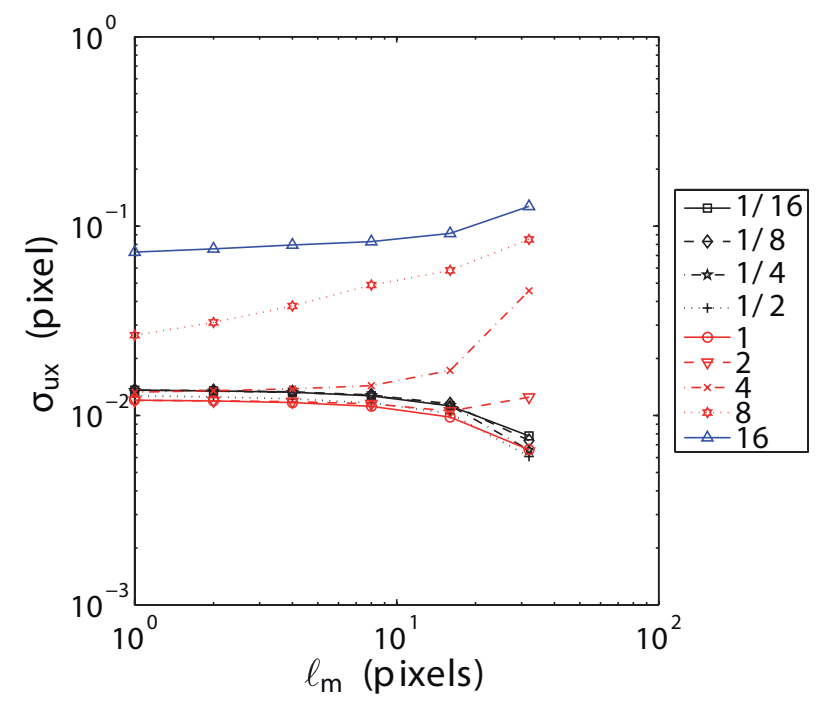

Figure 3: Change of the standard displacement error for different regularization lengths and different $\ell_{b} / \ell_{m}$ ratios. For each curve, the relaxation process is to be read from right to left. 


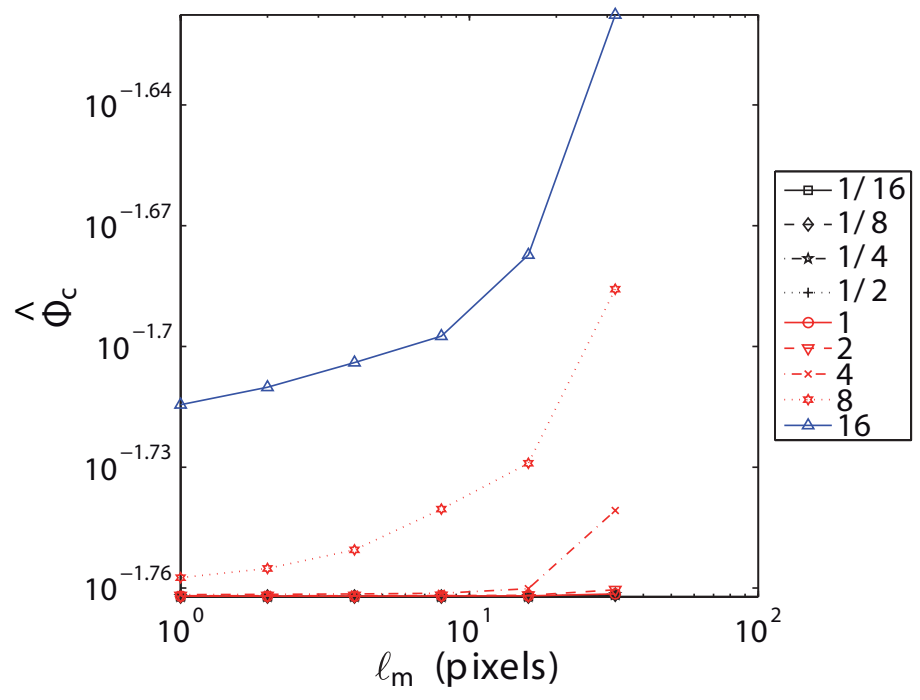

Figure 4: Change of the dimensionless correlation residual for different regularization lengths and different $\ell_{b} / \ell_{m}$ ratios. For each curve, the relaxation process is to be read from right to left. 


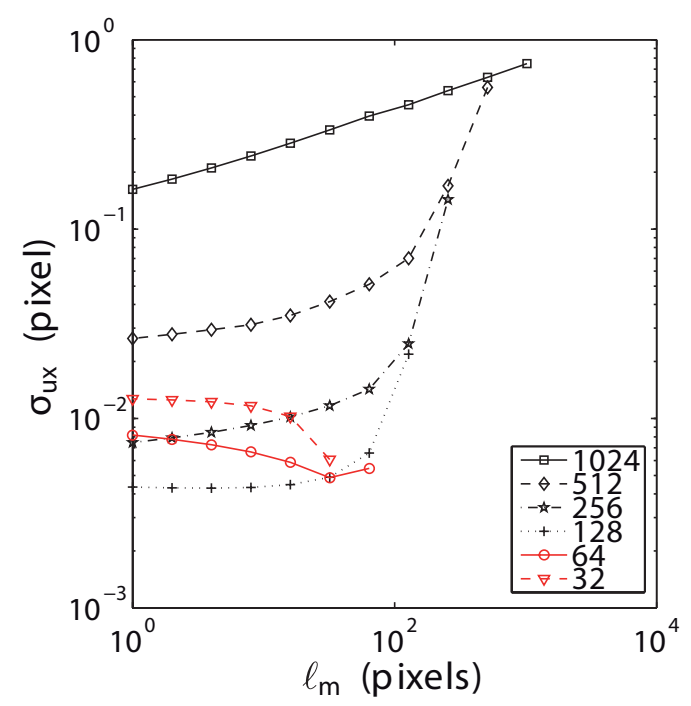

(a)

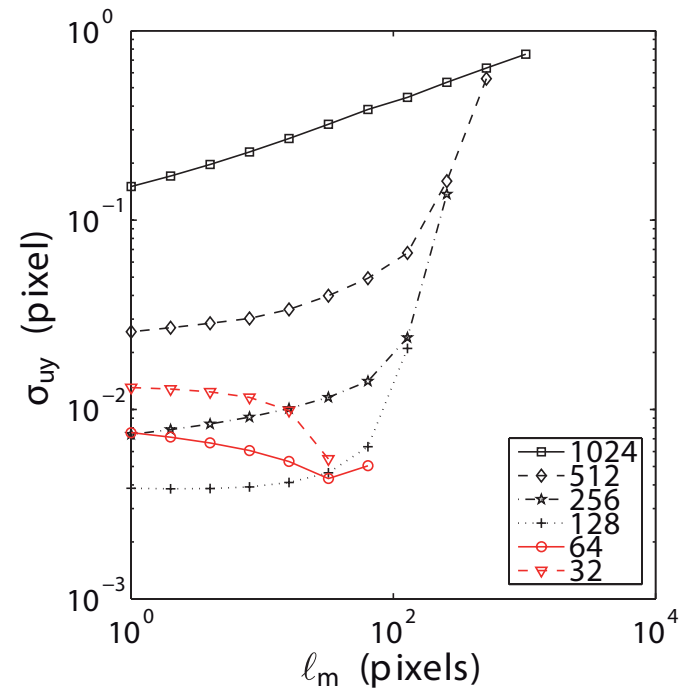

(b)

Figure 5: Change of the standard displacement error for different regularization lengths and different initialization lengths $\ell_{m}$ (in pixels) indicated in the legend. 


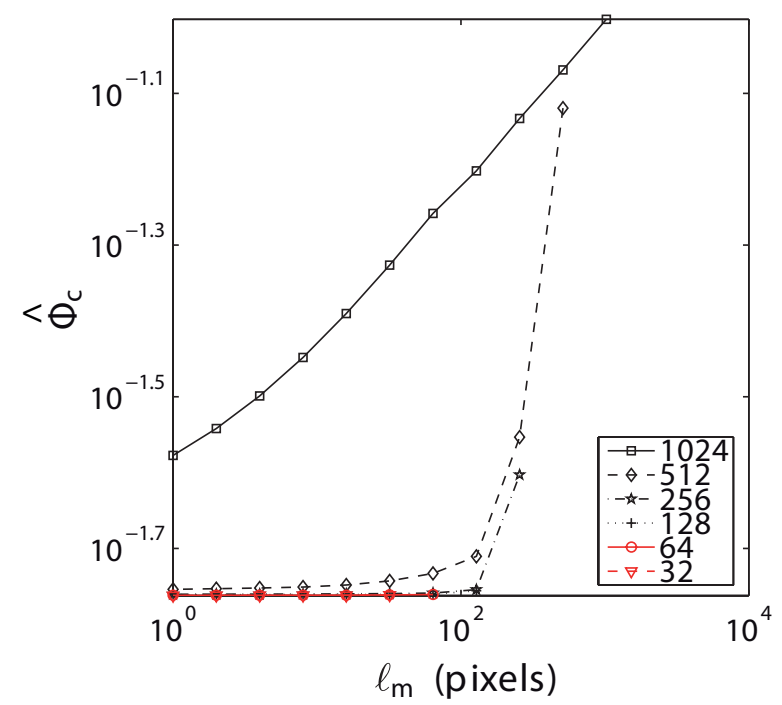

(a)

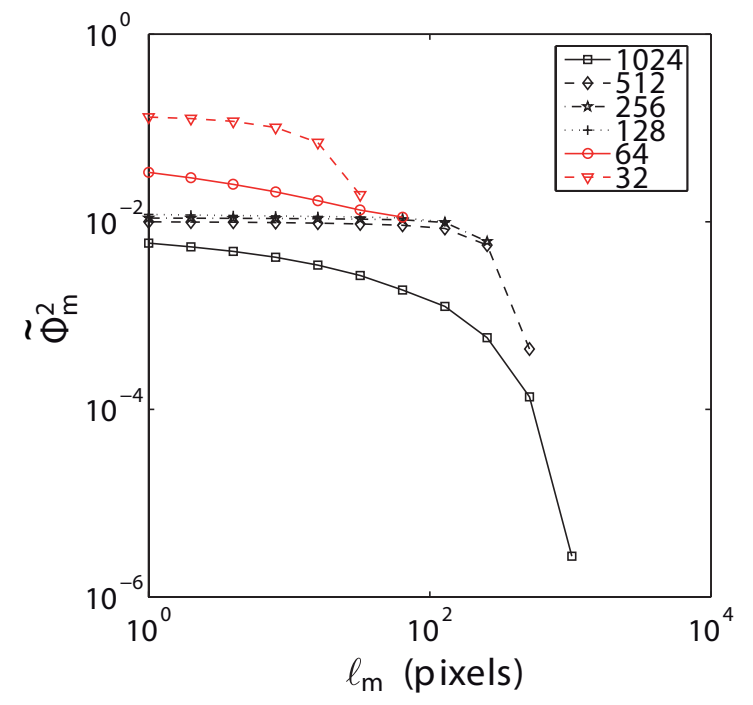

(b)

Figure 6: Change of the dimensionless correlation residuals (a) and equilibrium residuals (b) for different regularization lengths and different initialization lengths $\ell_{m}$ (in pixels) indicated in the legend. 


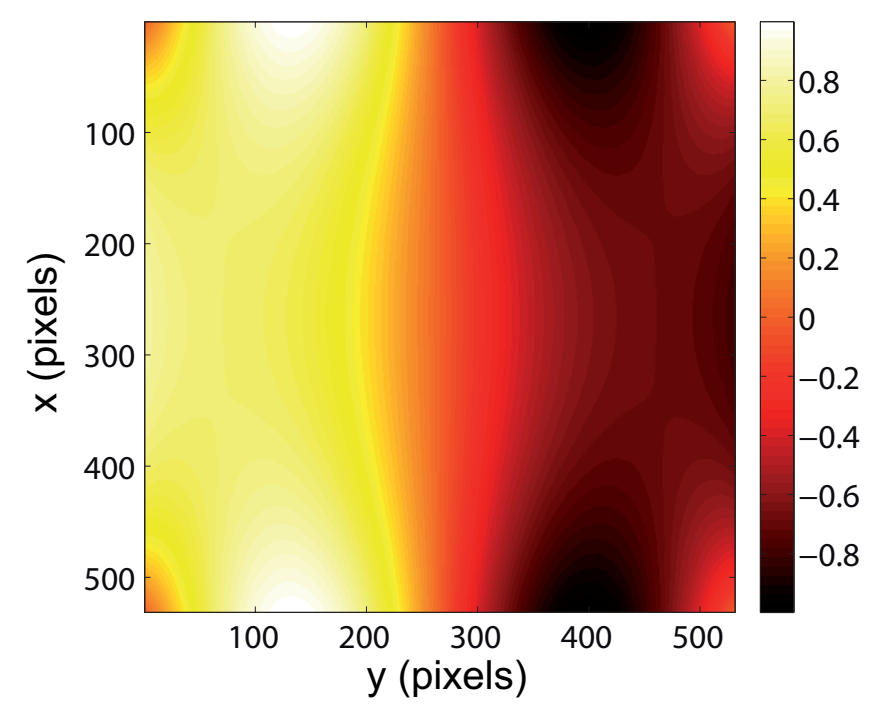

(a)

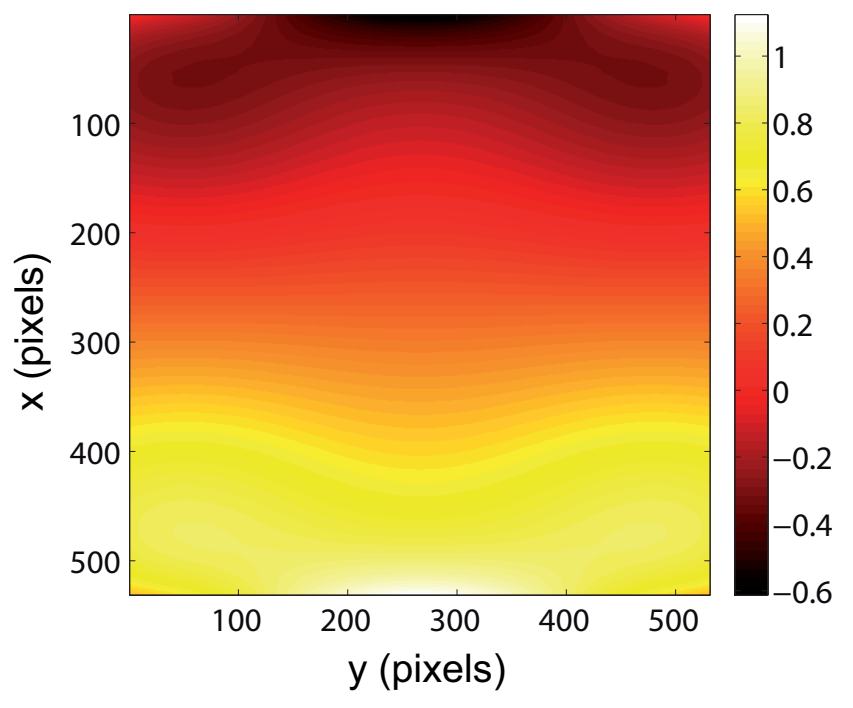

(b)

Figure 7: Prescribed displacement field in the mechanical test case in the vertical (a) and horizontal (b) directions. The displacements are expressed in pixels. 


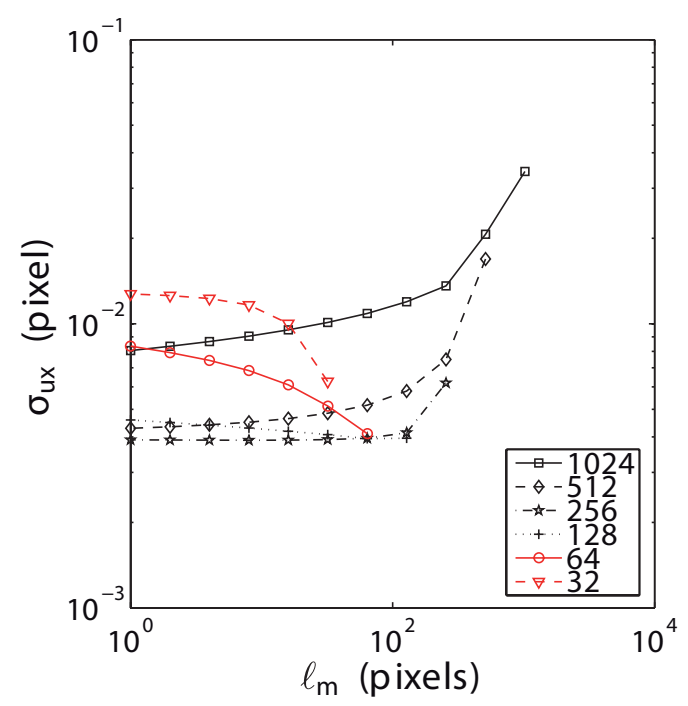

(a)

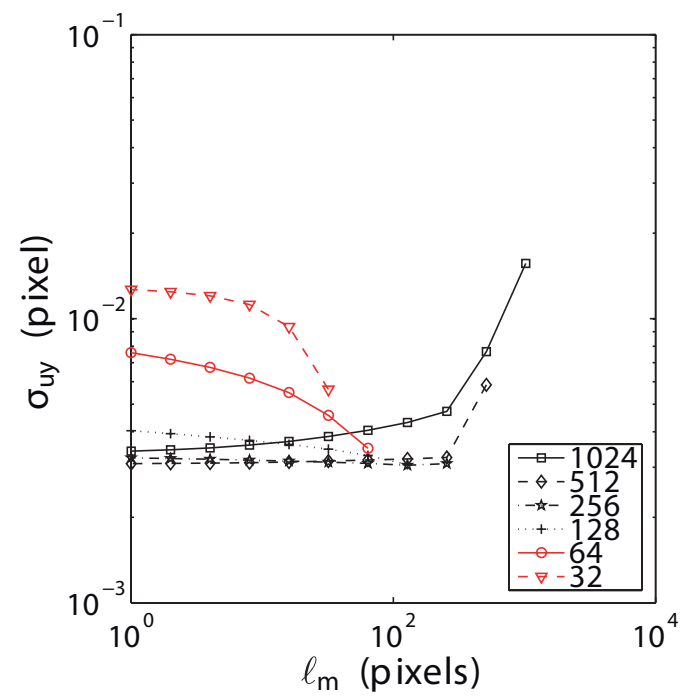

(b)

Figure 8: Change of the standard displacement error for different regularization lengths and different initialization lengths $\ell_{m}$ (in pixels) indicated in the legend. 


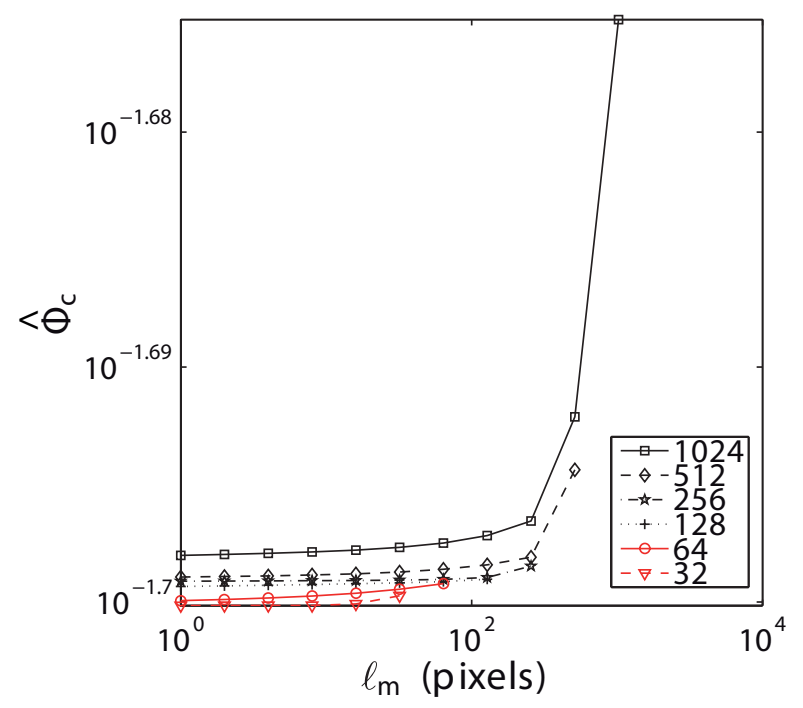

(a)

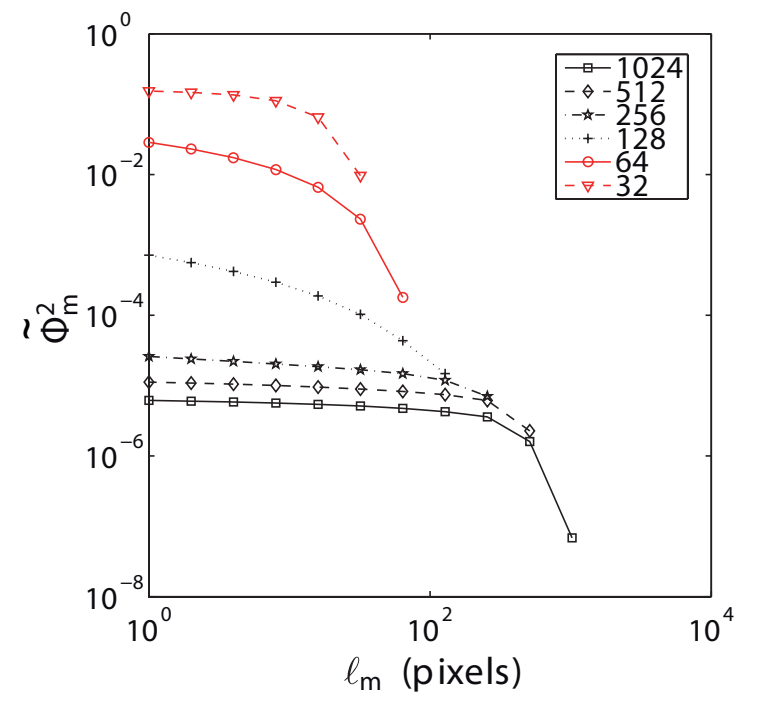

(b)

Figure 9: Change of the dimensionless correlation residuals (a) and equilibrium residuals (b) for different regularization lengths and different initialization lengths $\ell_{m}$ (in pixels) indicated in the legend. 


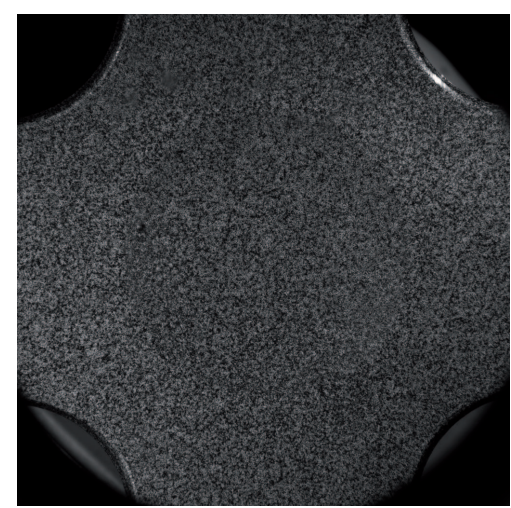

(a)

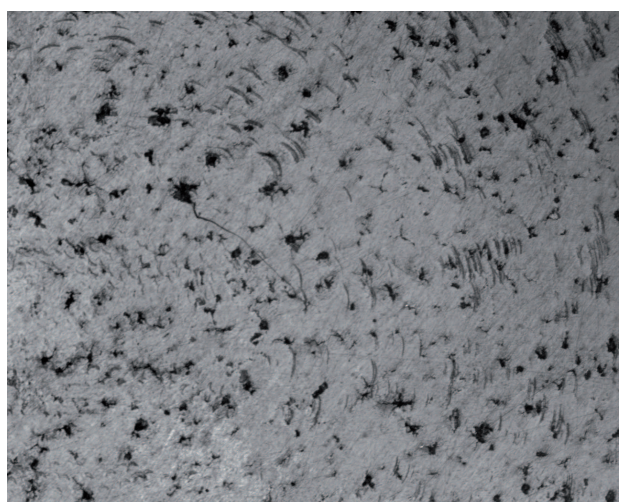

(b)

Figure 10: Reference pictures at the macroscopic (a) and mesoscopic (b) scales of a cross-shaped sample made of nodular graphite cast iron. 


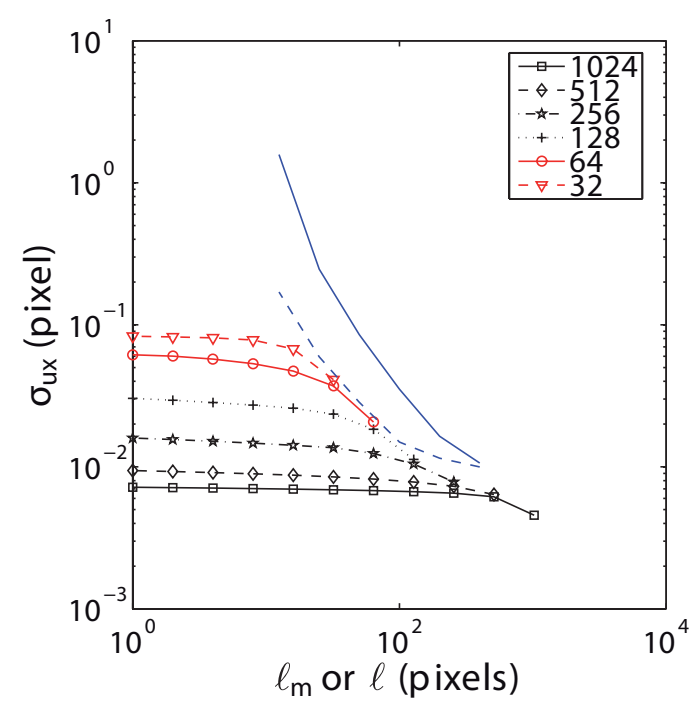

(a)

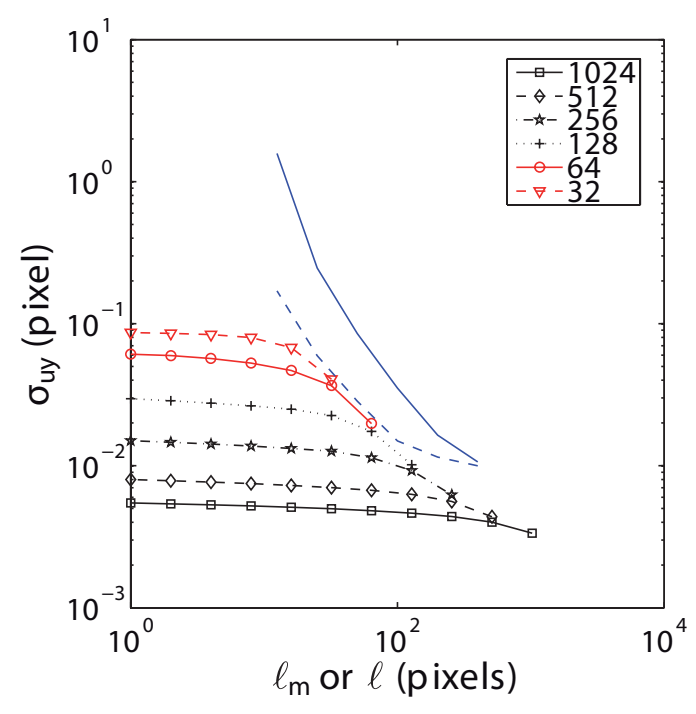

(b)

Figure 11: Standard displacement resolution in the vertical (a) and horizontal (b) directions for different regularization lengths $\ell_{m}$, ZOI (dashed blue line) or element (solid blue line) sizes $\ell$ when $\ell_{b} / \ell_{m}=1 / 2$. The legend indicates the initial length $\ell_{m}$ (in pixels). 


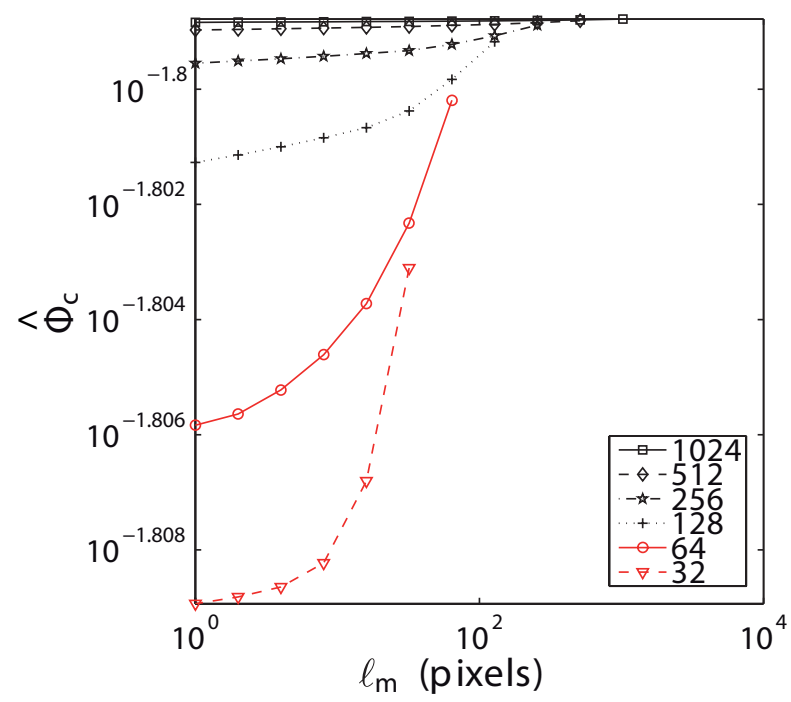

(a)

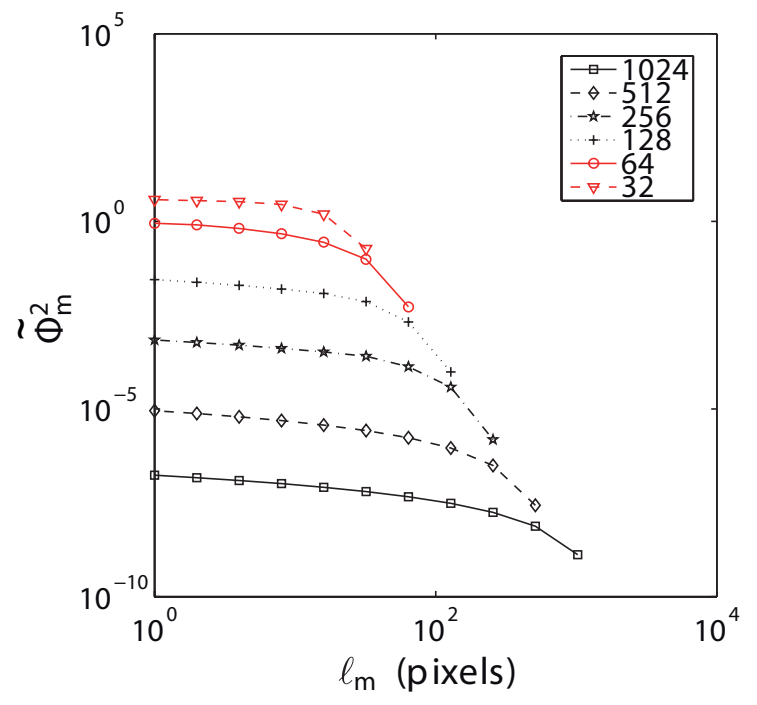

(b)

Figure 12: Change of the dimensionless correlation residuals (a) and equilibrium residuals (b) for different regularization lengths and different initializations. Note the narrow range of variation of $\hat{\Phi}_{c}$. 


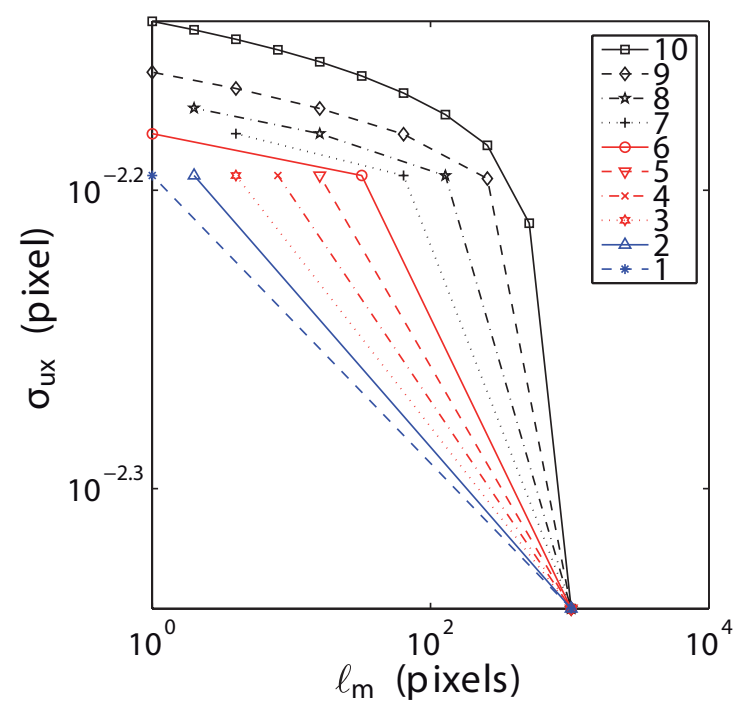

(a)

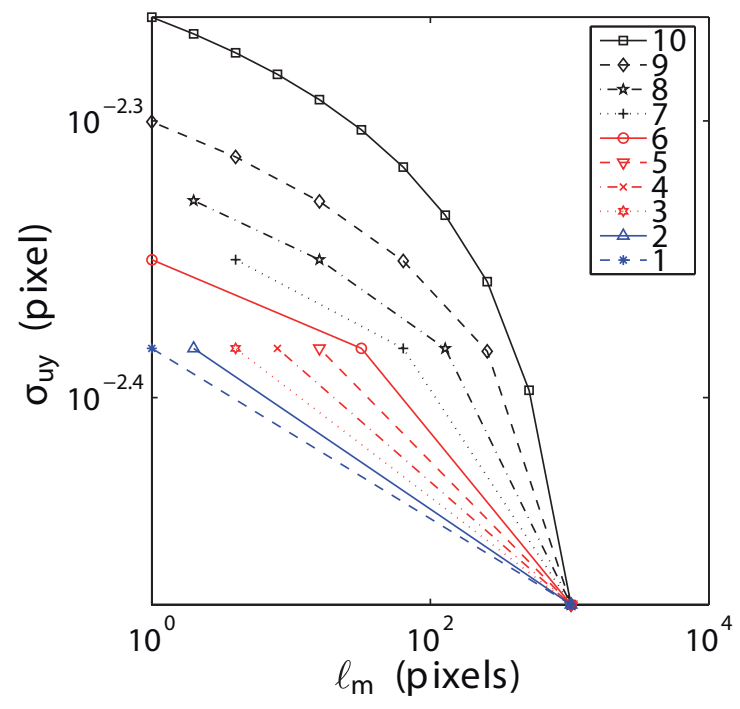

(b)

Figure 13: Standard displacement resolution in the vertical (a) and horizontal (b) directions for different regularization lengths and different number of jumps of the regularization length (indicated in the legend). 


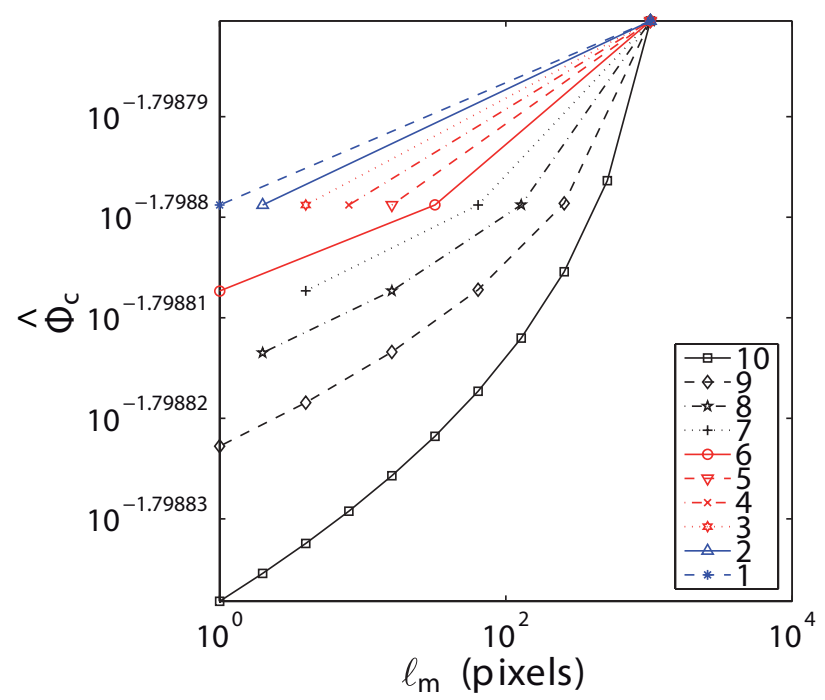

(a)

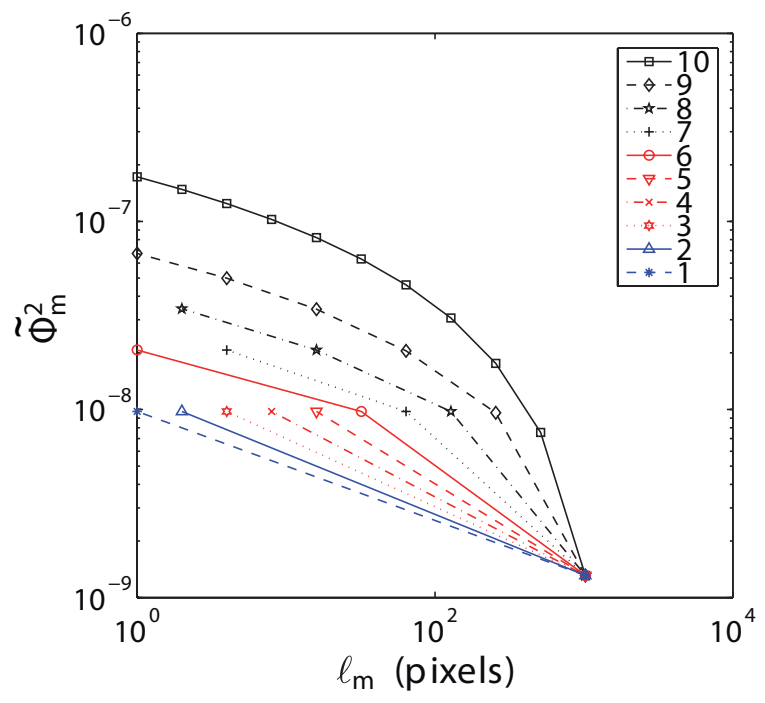

(b)

Figure 14: Change of the dimensionless correlation residuals (a) and equilibrium residuals (b) for different regularization lengths and and different number of jumps (indicated in the legend) to decrease the regularization length. Note the narrow range of variation of $\hat{\Phi}_{c}$. 


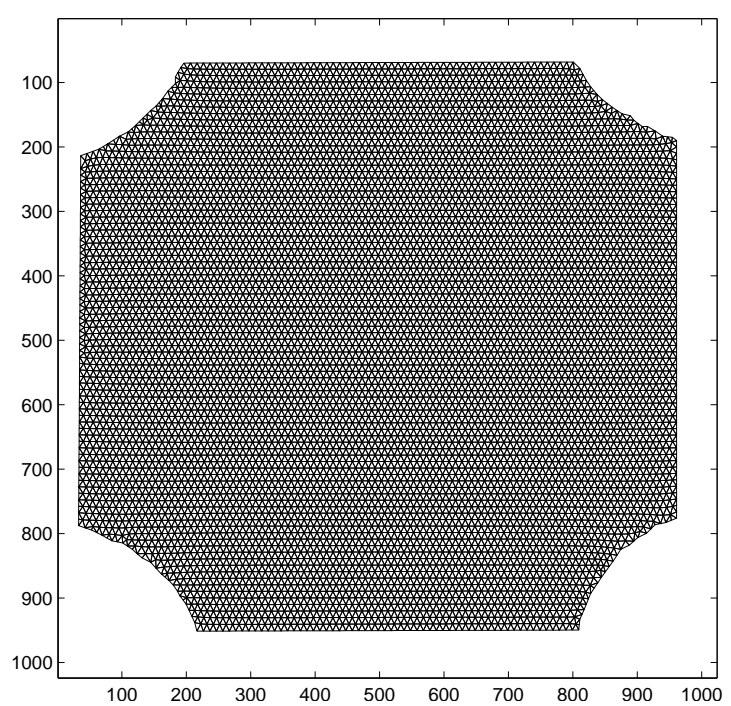

Figure 15: Mesh made of T3 elements used in the MA-DIC analyses at the macroscopic scale (see Figure 10(a)). 


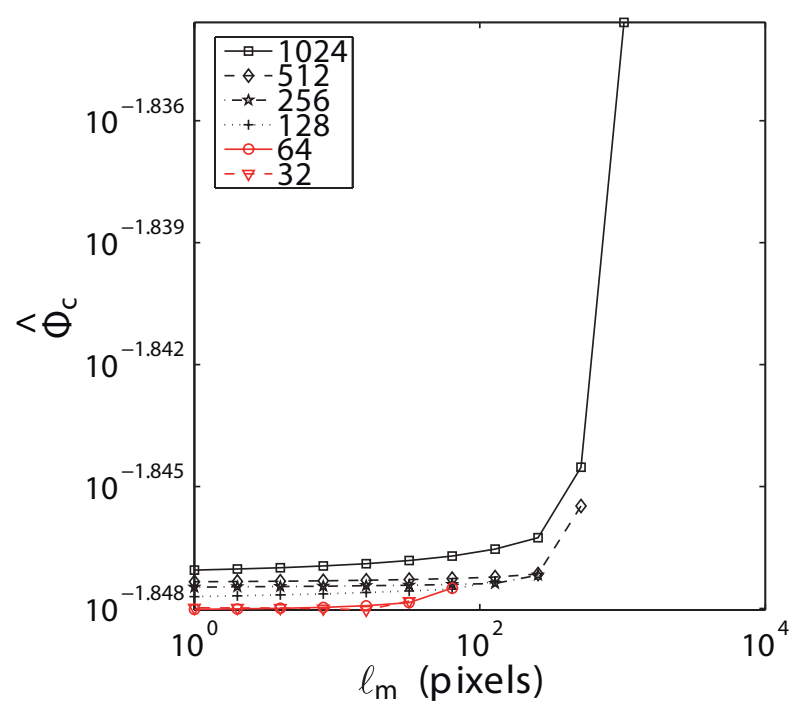

(a)

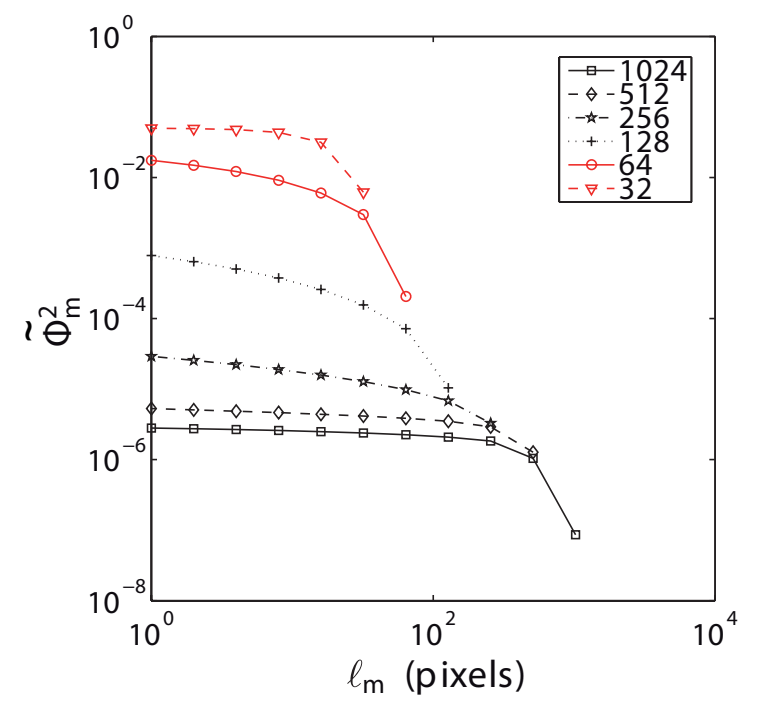

(b)

Figure 16: Change of the dimensionless correlation residuals (a) and equilibrium residuals (b) for different regularization lengths and different initialization lengths $\ell_{m}$ (in pixels) indicated in the legend. 

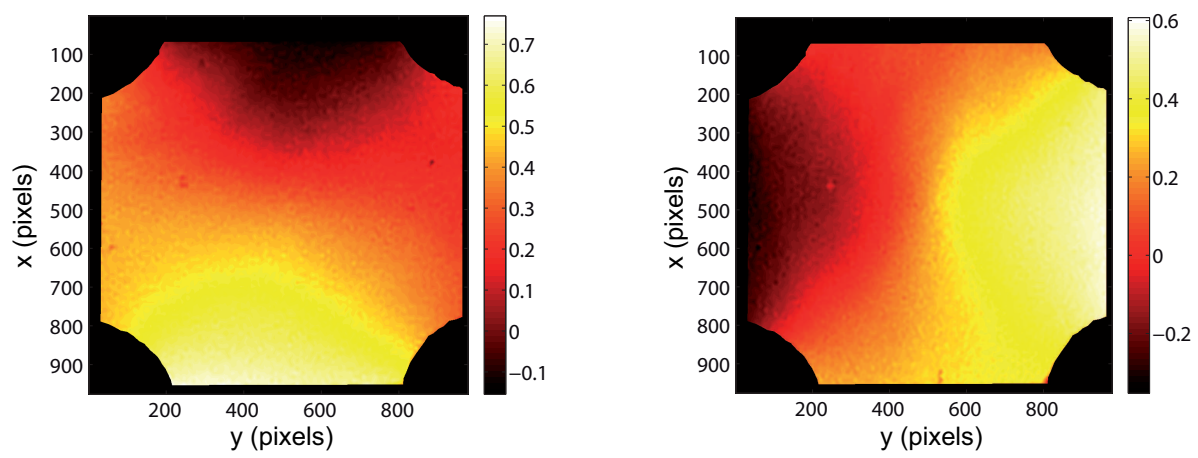

(a) $\ell_{m}=32$ pixels
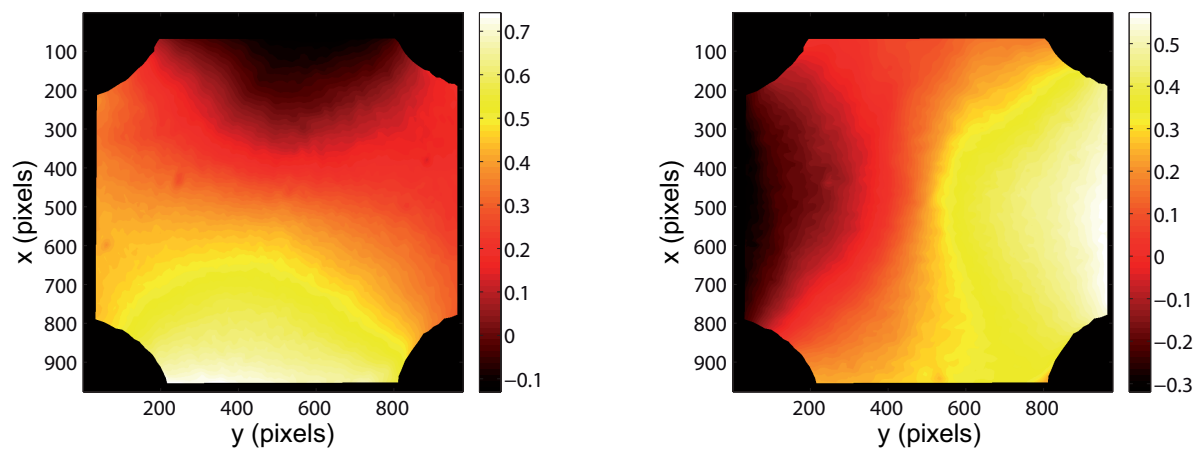

(b) $\ell_{m}=128$ pixels
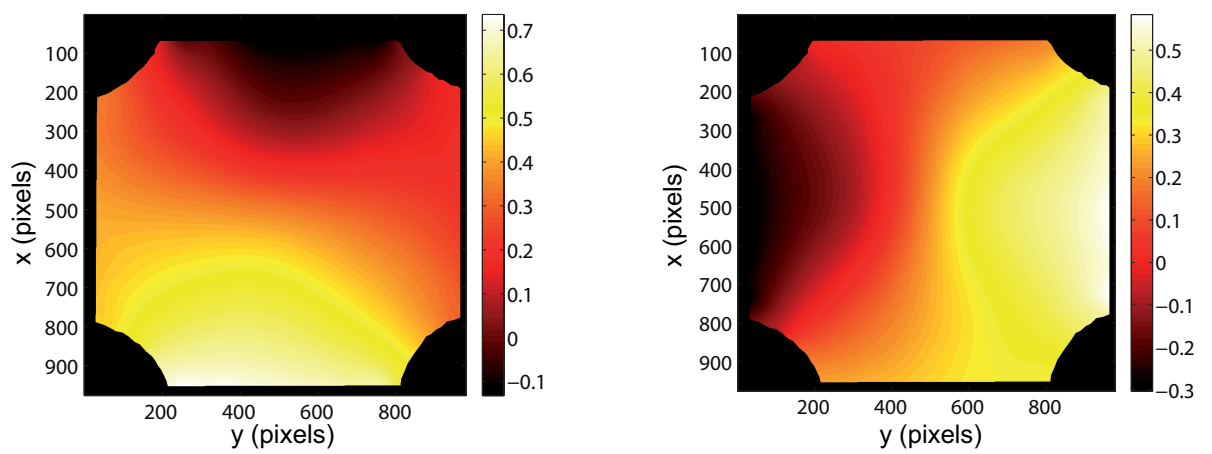

(c) $\ell_{m}=1024$ pixels

Figure 17: Measured displacement field in the biaxial test in the vertical (left) and horizontal (right) directions. Three initial regularization lengths $\ell_{m}$ are considered. The final regularization length is identical $\left(\ell_{m}=1\right.$ pixel $)$. The displacements are expressed in pixels. 